Noname manuscript No.

(will be inserted by the editor)

\title{
A New Spread Estimator
}

\section{Michael Bleaney · Zhiyong Li}

Abstract A new estimator of bid-ask spreads is presented. When the trade direction is known, any estimate of the spread is associated with a unique series of conjectural mid-prices derived by adjusting the observed transaction price by half the estimated spread. It is shown that the covariance of successive conjectural mid-price returns is maximised (or least negative) when the estimated spread is equal to the true spread. A search procedure to maximise this covariance may therefore be used to estimate the true spread. The performance of this estimator under various conditions is examined both theoretically and with Monte Carlo simulations. The simulations confirm the theoretical results. The performance of the estimator is good.

Keywords Bid-ask Spread, Feedback Trading, Estimation

JEL Classification C15 - G20

Michael Bleaney, Professor of Economics

University of Nottingham

School of Economics, University of Nottingham, University Park, Nottingham NG7 2RD, England,UK

Tel.: +441159515464

Fax +44 (0) 1159514159

E-mail: michael.bleaney@nottingham.ac.uk

Zhiyong Li

University of Nottingham

Room 268, Administration Building, Nottingham University Business School, The Univer-

sity of Nottingham, 199 Taikang East Road, Ningbo 315100 China

Tel: +8657488186465

Fax: +865748818 0125

E-mail: Zhiyong.Li@nottingham.edu.cn 
Noname manuscript No.

(will be inserted by the editor)

\begin{abstract}
A new estimator of bid-ask spreads is presented. When the trade direction is known, any estimate of the spread is associated with a unique series of conjectural mid-prices derived by adjusting the observed transaction price by half the estimated spread. It is shown that the covariance of successive conjectural mid-price returns is maximised (or least negative) when the estimated spread is equal to the true spread. A search procedure to maximise this covariance may therefore be used to estimate the true spread. The performance of this estimator under various conditions is examined both theoretically and with Monte Carlo simulations. The simulations confirm the theoretical results. The performance of the estimator is good.
\end{abstract}

Keywords Bid-ask Spread, Feedback Trading, Estimation JEL Classification C15 · G20

\title{
1 Introduction
}

Bid-ask spreads are important measures of liquidity in financial markets. Developing efficient spread estimators has been a lively area of research for several decades. The development of electronic trading in the major markets has reduced spreads and made them more transparent, since quoted spreads are often recorded. Nevertheless spread estimation is still important for less liquid markets and where "price improvement" occurs, i.e. where the trader obtains a better price for the transaction than that quoted to them.

Bid-ask spread estimators can be divided into two groups: the Roll family of estimators which are based on the serial correlation of transaction returns (Roll 1984, Glosten and Harris 1988, Choi et al. 1988, Stoll 1989, George et al. 1991, Laux and Senchack 1994, Huang and Stoll 1997 and Hasbrouck 2004, 2009), and other estimators such as Lesmond et al. (1999), Holden (2009), Goyenko et al. (2009) and Corwin and Schultz (2012).

One issue that has received little attention is the effect on spread estimators of feedback trading (order flows reacting to price returns). Feedback trading has been empirically recorded in stock markets by Hasbrouck (1991) and Nofsinger and Sias (1999), in futures markets by Lin et al. (2005), in foreign exchange markets by Daníelsson and Love (2006) and in bond markets by Dean and Faff (2008). Time aggregation of data can artificially introduce apparent feedback trading if order flows drive returns, as Daníelsson and Love (2006) point out. De Long et al. (1990) provide a theoretical model of feedback trading. Huang and Stoll's (1997) estimator is biased in the presence of feedback trading, as is the entire family of Roll-type estimators (Bleaney and Li 2015).

In this essay we introduce a new trial-and-error method of estimating the spread that has the same data requirements as the Huang and Stoll 1997 model (the HS model thereafter) in that it uses trade direction as well as return information, but which performs better than the HS model in certain circumstances, and specifically in the presence of feedback trading. When transaction prices and the direction of transactions are known, any estimate of the spread is associated with an estimate of the mid-price for each transaction. The proposed method relies on the fact that, under certain assumptions, the estimated covariance of current and lagged mid-price returns will be maximized (or least negative) when 
the estimated spread is equal to the true spread. A search to maximize this estimated covariance should therefore yield an accurate estimate of the spread. The assumptions are that mid-price returns are independent of past, current and future order flows. This happens if order flows do not react to returns (no feedback trading), and returns do not react to order flows (no price adjustment by dealers for inventory control or precautionary reasons). As with the HS estimator, information on transaction data and order flows is needed. Like the HS estimator, our new estimator is biased in the presence of feedback trading, but substantially less biased than the HS estimator.

When order flows are unknown, one may apply the method introduced in Hasbrouck $(2004,2009)$ to obtain order flows before using our estimator, as is the case with the HS estimator. The combination of our new estimator and Hasbrouck's method should generate satisfactory estimates.

The rest of the paper is organised as follows. Section Two introduces a new estimator based on conjectures about the spread. Section Three discusses the possible biases of this estimator under the existence of feedback trading and the price impact of orders. Section Four compares the performance of the new estimator with that of the Roll, Huang-Stoll and Corwin-Schultz estimators using simulation evidence for sampling frequencies ranging from one minute to 24 hours. The simulations show the average estimation error as well as the bias of each estimator for each experiment. Section Five discusses the results of simulations. Section Six concludes.

\section{A New Estimator}

In this section, we introduce a new estimator based on conjectures about the spread. We show that under ideal conditions the new estimator is unbiased.

The intuition is simple. We make a conjecture about the spread and calculate conjectural mid-price returns according to the conjecture. The conjectural error (the difference between the conjecture and the true spread) influences the covariance between two adjacent conjectural mid-price returns. It will be shown that the series of true mid-price returns has the greatest covariance among all other series of conjectural mid-price returns. Therefore, after trying conjectural spreads and calculating the corresponding conjectural mid-price returns and the covariances, we use the conjectural spreads which correspond to the series with the greatest covariance as the estimate of the true spread.

The bid-ask spread is the difference between the ask price and the bid price. Let $s_{t}$ be the transaction price which is the ask (bid) price if a buy (sell) order is executed. Transaction prices can be divided into two parts. One is the bid-ask spread and the other is the unobserved mid-price. Formally, the price is given by:

$$
s_{t}=M_{t}+\frac{S P}{2} \cdot B S_{t}
$$

where $M_{t}$ is the mid-price. $S P$ is the effective bid-ask spread, and $B S$ is the trade indicator which shows the direction of the trade. $B S=1$ if there is a buy order and $B S=-1$ if there is a sell order. Then the transaction price return is given by:

$$
\Delta s_{t}=\Delta M_{t}+\frac{S P}{2}\left(B S_{t}-B S_{t-1}\right)
$$

where $\Delta$ is the first order difference operator. The spread will enlarge (reduce) the observed return when the change in the trade direction has the same (opposite) sign as the mid-price change. If the trade direction does not change $\left(B S_{t}-B S_{t-1}=0\right)$, the observed return is equal to the mid-price change.

We assume that returns are uncorrelated with past, current or future order flows. This means that there is no feedback trading (order flows do not react to past returns), and no adjustment of prices to order flows for inventory control or precautionary reasons. These are the ideal conditions for the estimator. If we have a conjecture about the spread, the error of the conjecture is given by:

$$
\Omega_{t}=S P_{t}-\widetilde{S P}_{t}
$$

where $\widetilde{S P}_{t}$ is the conjecture, and $\Omega_{t}$ is the conjectural error. All the symbols with $\sim$ represent conjecture values.

We assume that the spread is fixed throughout the series. Thus the spread and its conjecture and the conjectural error are fixed.

$$
\Omega=S P-\widetilde{S P}
$$


A conjectural mid-price series $\left(\widetilde{M}_{t}\right)$ can be obtained from conjectural spreads.

$$
\widetilde{M}_{t}=s_{t}-\frac{1}{2} \widetilde{S P B} S_{t}
$$

If we re-arrange the equation above, one can find that the difference between the true mid-price and its conjecture is half the conjectural error:

$$
\begin{aligned}
\widetilde{M}_{t} & =M_{t}+\frac{1}{2} S P B S_{t}-\frac{1}{2} \widetilde{S P} B S_{t} \\
& =M_{t}+\frac{1}{2} B S_{t}(S P-\widetilde{S P}) \\
& =M_{t}+\frac{1}{2} B S_{t} \Omega
\end{aligned}
$$

Then the mid-price return is given by:

$$
\Delta \widetilde{M}_{t}=\Delta M_{t}+\frac{1}{2} \Omega B S_{t}-\frac{1}{2} \Omega B S_{t-1}
$$

We now show that under ideal conditions a trial-and-error method can identify the true spread.

Definition 1 Let $A$ be a set of all conjectures of the true spread $A=\left\{\widetilde{S P}_{1}, \widetilde{S P}_{2}, \ldots, \widetilde{S P}_{n}\right\}$

Definition 2 Let B be a set of covariances of two adjacent conjectural mid-price returns obtained according to the conjecture of the true spread $B=\left\{\operatorname{Cov}_{1}, \operatorname{Cov}_{2}, \cdots, \operatorname{Cov}_{n}\right\}$, where $\operatorname{Cov}_{i}=\operatorname{Cov}\left[\widetilde{M}\left(\widetilde{S P_{i}}\right)_{t}, \widetilde{M}\left(\widetilde{S P_{i}}\right)_{t-1}\right]$.

Proposition 1 If there is no feedback trading, and no inventory control or asymmetric information components of the spread, then the spread and its conjecture, and thus the conjectural error, are serially independent or are fixed. If a conjecture of the spread $\widetilde{S P}_{i} \in A$ corresponds to $\operatorname{Cov}_{i}=\max (B)$, it equals the true spread i.e. $\widetilde{S P}_{i}=S P$.

Proof The full proof is in the appendix. The covariance of two adjacent conjectures of mid-price returns is:

$$
\begin{aligned}
& \operatorname{Cov}\left(\Delta \tilde{M}_{t}, \Delta \tilde{M}_{t-1}\right) \\
= & E\left\{\left[\Delta \widetilde{M}_{t}-E\left(\Delta \widetilde{M}_{t}\right)\right]\left[\Delta \widetilde{M}_{t-1}-E\left(\Delta \widetilde{M}_{t-1}\right)\right]\right\}
\end{aligned}
$$

Assume the expectation of the conjectural mid-prices is zero. Thus, the equation above can be written as:

$$
\begin{aligned}
& \operatorname{Cov}\left(\Delta \widetilde{M}_{t}, \Delta \widetilde{M}_{t-1}\right) \\
= & E\left[\Delta \widetilde{M}_{t} \cdot \Delta \widetilde{M}_{t-1}\right] \\
= & E\left[\left(\Delta M_{t}+\frac{1}{2} \Omega B S_{t}-\frac{1}{2} \Omega B S_{t-1}\right)\left(\Delta M_{t-1}+\frac{1}{2} \Omega B S_{t-1}-\frac{1}{2} \Omega B S_{t-2}\right)\right]
\end{aligned}
$$

As shown in the Appendix, the assumptions imply that $B S$ is independent of $\Delta M$ at all dates, so many terms in (9) are zeros. The variable $B S$ is a binary variable $(1$ or -1$)$, thus $E\left(B S_{t-1}^{2}\right)=1$. Then we can finally obtain:

$$
\begin{aligned}
& \operatorname{Cov}\left(\Delta \tilde{M}_{t}, \Delta \tilde{M}_{t-1}\right) \\
= & \operatorname{Cov}\left(\Delta M_{t} \cdot \Delta M_{t-1}\right)+\frac{1}{4} \Omega^{2} \cdot\left[2 E\left(B S_{t} \cdot B S_{t-1}\right)-E\left(B S_{t} \cdot B S_{t-2}\right)-1\right]
\end{aligned}
$$

The right hand side of the equation is a quadratic polynomial of the expectation of the error of the conjecture. For a given series, the first term on the right hand side is a constant. It is straightforward that when the error is zero (i.e. $\Omega=0$ ), the second term is zero. Furthermore, when $\Omega=0$, there is a global extreme for the right hand side polynomial, symmetrically, the left hand side of the equation $\operatorname{Cov}\left(\Delta \widetilde{M}_{t}, \Delta \widetilde{M}_{t-1}\right)$ is also at the extreme value:

$$
\underset{\Omega}{\arg \max } \operatorname{Cov}\left(\Delta \tilde{M}_{t}, \Delta \widetilde{M}_{t-1}\right)=0
$$

When the conjectural error is zero, the conjectural spread is the true spread:

$$
\Omega=S P-\widetilde{S P}_{i}=0
$$

Therefore the conjectural spread which maximises the covariance equals the true spread.

$$
\underset{\widetilde{S P}_{i} \in A}{\arg \max } \operatorname{Cov}\left(\Delta \tilde{M}_{t}, \Delta \widetilde{M}_{t-1}\right)=S P
$$

Q.E.D. 
Proposition 1 sheds light on the spread estimation. The most important point is that at the extreme point, the expectation of conjectural spread equals that of the true spread. Therefore, one can apply a search procedure to estimate the true spread.

More specifically, the first step of the estimation is to choose a conjectural spread $\left(\widetilde{S P}_{i}\right)$. Secondly, calculate the conjectural mid-prices $\left(\widetilde{M}_{i}\right)$, and then the conjectural returns $\left(\Delta \widetilde{M}_{t, i}\right)$ using the conjectural spread according to Equation (5). Thirdly, calculate the covariance of two adjacent returns of conjectural mid-prices $\left(\operatorname{Cov}\left(\Delta \widetilde{M}_{t, i}, \Delta \widetilde{M}_{t-1, i}\right)_{i}\right)$. Fourthly, repeat the first three steps enough times to draw a curve of the covariance against the conjectural spread. Finally, find the maximum point of the curve and the conjectural spread corresponding to the maximum point is the estimate of the spread. Because the curve is a negative parabola, there is no need to try all possible values of the spread, instead, one can stop when the shape of a negative parabola appears.

Figure (1) presents the intuition underlying Proposition (1) for the simple case where the true midprice does not change over three periods. It shows the general relationships between the returns of true mid-prices and the returns of conjectural mid-prices, when only transaction prices and the direction of transactions are known. The conjectural spread here is less than the true spread $(\omega>0)$. The conjectural mid-prices are obtained from Equation (5) using transaction prices, given a conjectural spread. The dotted lines are conjectural mid-prices and the solid lines are true mid-prices or transaction prices. $A$ and $B$ represent the ask and bid prices respectively; these are the prices that are actually observed. $M$ and $\widetilde{M}$ represent the true mid-price and the conjecture of it respectively. $\Delta$ is the first-order difference operator. $\Omega$ is the error of the conjecture which is the difference between the true spread and the conjectural spread, or equivalently, between the true mid-price and the conjectural mid-price.

There is a sell order in period $t-2$, and, thus, the bid price is recorded. There is a buy order in period $t-1$, and thus the ask price is recorded. There is a sell order in period $t$, and thus the bid price is recorded. Because the conjectural spread is less than the true spread, in periods $t-2$ and $t$, the conjectural mid-prices are $0.5 \cdot \Omega$ less than the true ones, and in period $t-1$, the conjectural mid-price is $0.5 \cdot \Omega$ greater than the true one.

Between periods $t-2$ and $t-1$, the trade direction changes from selling to buying, so the conjectural error makes the conjectural mid-price return greater than the true mid-price return $\left(\Delta \widetilde{M}_{t-1}=\Delta M_{t-1}+\right.$ $\Omega=\Omega$ ).

Between periods $t-1$ and $t$, the trade direction switches back from buying to selling, so the conjectural error partially cancels the true mid-price return $\left(\Delta \widetilde{M}_{t}=\Delta M_{t}-\Omega=-\Omega\right)$. When the trade direction does not change, the returns of the conjectural mid-prices and of the true mid-prices are the same.

In the case shown in Figure (1), the covariance of two adjacent returns of true mid-prices is zero because the mid-price is fixed. The covariance of two adjacent conjectural mid-price returns is, however, negative. This is because, when the spread is underestimated, the conjectural mid-price returns take on some of the negative serial correlation of the transaction price series induced by the spread (as in Roll's (1984) analysis of the effect of the spread on the serial covariance of transaction price returns, the cases where the trade direction does not switch make no difference).

Now consider the opposite case where the spread is over-estimated (but true mid-price returns are still zero as in Figure 1). Then the conjectural mid-price would be below the true mid-price in periods $t-2$ and $t$, and above it in period $t-1$, so in this case also the conjectural mid-price series has negative serial covariance that is not present in the true mid-price series.

\section{Errors of the New Estimator}

\subsection{Feedback Trading and the New Estimator}

In this section, we discuss the impact of feedback trading on the performance of the estimator.

We assume that the mid-price returns can be written as follows:

$$
\Delta M_{t}=\epsilon_{t}
$$

where $\epsilon_{t}$ is a shock which is not influenced by order flows. 


\section{Fig. 1 The Conjecture of the Spread}

The figure show the general relationships between the returns of true mid-prices and the returns of conjectural mid-prices, when only transaction prices and the directions of transactions are known. The conjectural spread here is less than the true spread. The conjectural mid-prices are obtained from Equation (5) using transaction prices, given a conjectural spread. The dotted lines are conjectural mid-prices and the solid lines are true midprices or transaction prices. $A$ and $B$ represent the ask and bid prices respectively; these are the prices that are actually observed. $M$ and $\widetilde{M}$ represent the true mid-price and the conjecture of it respectively. $\Delta$ is the first-order difference operator. $\Omega$ is the error of the conjecture which is the difference between the true spread and the conjectural spread, or equivalently, between the true mid-price and the conjectural mid-price.

There is a sell order in period $t-2$, and, thus, the bid price is recorded. There is a buy order in period $t-1$, and thus the ask price is recorded. There is a sell order in period $t$, and thus the bid price is recorded. Because the conjectural spread is less than the true spread, the in periods $t-2$ and $t$, the conjectural mid-prices are $0.5 \cdot \Omega$ less than the true ones, and in period $t-1$, the conjectural mid-price is $0.5 \cdot \Omega$ greater than the true one.

Between periods $t-2$ and $t-1$, the trade direction changes from selling to buying, so the conjectural error makes the conjectural mid-price return greater than the true mid-price return $\left(\Delta \widetilde{M}_{t-1}=\Delta M_{t-1}+\Omega=\Omega\right)$.

Between periods $t-1$ and $t$, the trade direction switches back from buying to selling, so the conjectural error partially cancels the true mid-price return $\left(\Delta \widetilde{M}_{t}=\Delta M_{t}-\Omega=-\Omega\right)$. When the trade direction does not change, the returns of the conjectural mid-prices and of the true mid-prices are the same.

If feedback trading exists, order flows are influenced by the shocks. Formally, the covariance between order flows and the shocks is not zero:

$$
\begin{aligned}
& \operatorname{Cov}\left(\epsilon_{t-1} \cdot B S_{t-1}\right)=E\left(\epsilon_{t-1} \cdot B S_{t-1}\right) \neq 0 \\
& \operatorname{Cov}\left(\epsilon_{t-1} \cdot B S_{t}\right)=E\left(\epsilon_{t-1} \cdot B S_{t}\right) \neq 0
\end{aligned}
$$

We assume a quote-driven market in which traders receive price quotes, and therefore observe the shock $\left(\epsilon_{t-1}\right)$ before placing the order $\left(B S_{t-1}\right)$. Then we define the covariance between $\epsilon_{t-1}$ and $B S_{t-1}$ as current feedback trading (one-period feedback trading). The influence of the shock may persist in the next period, i.e. the shock $\epsilon_{t-1}$ may influence the order flow in period $t$ as well. We define the covariance between $\epsilon_{t-1}$ and $B S_{t}$ as lagged feedback trading (two-period feedback trading).

Proposition 2 If there is feedback trading (equations 15 are valid), and no inventory control or asymmetric information components of the spread, then the spread and its conjecture, and thus the conjectural error, are serially independent or are fixed. If a conjecture of the spread $\widetilde{S P}_{i} \in A$ corresponds to $\operatorname{Cov}_{i}=\max (B)$, then $\widetilde{S P}_{i}=S P+E\left(\epsilon_{t-1} \cdot B S_{t-1}\right)-E\left(\epsilon_{t-1} \cdot B S_{t}\right)$.

Proof With the existence of feedback trading, the covariance between two adjacent conjectures of midprice returns is (the detail of the deduction is presented in appendix B and C.1):

$$
\begin{aligned}
& \operatorname{Cov}\left(\Delta \widetilde{M}_{t}, \Delta \widetilde{M}_{t-1}\right) \\
= & E\left(\Delta \widetilde{M}_{t} \cdot \Delta \widetilde{M}_{t-1}\right) \\
= & E\left(\epsilon_{t} \cdot \epsilon_{t-1}\right)+\Pi_{1} \cdot \Omega^{2}+\Pi_{2} \cdot \Omega
\end{aligned}
$$

where

$$
\begin{aligned}
& \Pi_{1}=\frac{1}{4} E\left[2 B S_{t-1} \cdot B S_{t-2}-B S_{t} \cdot B S_{t-2}-1\right] \\
& \Pi_{2}=\frac{1}{2} E\left[-B S_{t-1} \cdot \epsilon_{t-1}+B S_{t} \cdot \epsilon_{t-1}\right]
\end{aligned}
$$

Equation (16) suggests that when there is feedback trading, the covariance of the conjectural mid-price returns $\left(\operatorname{Cov}\left(\Delta \widetilde{M}_{t}, \Delta \widetilde{M}_{t-1}\right)\right)$ contains a linear term in the conjectural errors $(\Omega)$ as well as a quadratic one, so the value of $\Omega$ which maximises the polynomial is no longer zero.In other words, the estimator is biased. 
We now discuss possible errors that arise if we still estimate the spread by maximising the covariance between two adjacent conjectures of mid-price returns $\operatorname{Cov}\left(\Delta \widetilde{M}_{t}, \Delta \widetilde{M}_{t-1}\right)$, as suggested in the previous section. The estimate is given by:

$$
\widehat{S P}=\left[\frac{2 \Pi_{1} S P+\Pi_{2}}{2 \Pi_{1}}\right]
$$

where $\widehat{S P}$ is obtained when $\Omega$ maximises $\operatorname{Cov}\left(\Delta \widetilde{M}_{t}, \Delta \widetilde{M}_{t-1}\right)$, and is the estimate of the true spread.

In the presence of feedback trading, the estimated spread should be (the detail of the deduction is presented in appendix C.1):

$$
\begin{aligned}
\widehat{S P} & =S P-4 \Pi_{2} \\
& =S P+E\left(\epsilon_{t-1} \cdot B S_{t-1}\right)-E\left(\epsilon_{t-1} \cdot B S_{t}\right)
\end{aligned}
$$

\section{Q.E.D.}

Equation (19) suggests the estimator will overestimate the spread if there is positive feedback trading and vice versa. It is of interest that, unlike the other estimators, the total influence of feedback trading on the estimator includes two period-feedback trading $\left(E\left(\epsilon_{t-1} \cdot B S_{t}\right)\right)$. If order flows do not exhibit serial autocorrelation, which may be because the influence of the mid-price shocks is persistent, there is only current feedback trading $\left(E\left(\epsilon_{t-1} \cdot B S_{t-1}\right)\right)$. One may define the difference between current and lagged feedback trading net feedback trading. Because the signs of current feedback trading and lagged feedback trading in Equation (19) are different, the positive autocorrelation of order flows can reduce the influence of feedback trading and vice versa. Because of hot-potato trading, order flows, especially in the tick-by-tick case, tend to be positively autocorrelated. Thus, the influence of net feedback trading on our estimator is not as big as on the others.

When there is no feedback trading $\left(\Pi_{2}=0\right)$, and no autocorrelated order flows $\left(E\left(B S_{t-1} \cdot B S_{t-2}\right)=0\right.$ and $\left.\Pi_{1}=-1\right)$, the equation above becomes:

$$
\widehat{S P}=S P
$$

and thus,

$$
\Omega=0
$$

The equations above suggest that under the ideal conditions, Equation (16) reduce to the simple version of the estimator and in this circumstance, the estimator is unbiased.

Consider now the impact of feedback trading in the HS model. The HS model is given by:

$$
\Delta s_{t}=\frac{S P}{2} B S_{t}+(\alpha+\beta-1) \frac{S P}{2} B S_{t-1}-\alpha \frac{S P}{2}(1-2 \theta) B S_{t-2}+\epsilon_{t}
$$

where $\alpha$ and $\beta$ represent the weights of the asymmetric information and inventory control components of the spread respectively. $\theta$ is the probability of order reversal. $\alpha, \beta$ and $\theta$ are concerning with the spread decomposition. In this paper, we focus on the coefficient of BSt, i.e. $\frac{\widehat{S P}}{2}$.

When there is feedback trading, the estimated value from the HS model $\frac{\widehat{S P}}{2}$ is as follows:

$$
\frac{\widehat{S P}}{2}=\frac{S P}{2}+E\left(B S_{t}, \epsilon_{t}\right)
$$

Then, the HS model error will be

$$
\text { Error }=2 \cdot E\left(B S_{t}, \epsilon_{t}\right)
$$

Equation (24) suggests that when there is positive feedback trading, the HS estimator overestimates the true spread and vice versa. In particular, when there is only current feedback trading, the bias in the HS estimator is twice as large as that of our new estimator. 
In this section, we discuss the impact of inventory holding costs (IC) and asymmetric information costs (AS) on the performance of the estimator. Under these conditions, the HS estimator performs well, because it explicitly incorporates these effects.

When there are IC\&AS components in the spread, the mid-price return is given by:

$$
\Delta M_{t}=\frac{1}{2} \varrho S P B S_{t-1}+\epsilon_{t}
$$

where mid-price returns are influenced by the past order flow (Evans and Lyons 2002), $\varrho$ is the fraction of the components of the spread and $\epsilon_{t}$ is a shock which is not influenced by order flows.

Proposition 3 If there is no feedback trading, and the mid-price is influenced by past trades, then the spread and its conjecture, and thus the conjectural error, are serially independent or are fixed. If a conjecture of the spread $\widetilde{S P}_{i} \in A$ corresponds to $\operatorname{Cov}_{i}=\max (B)$, then $\widetilde{S P}=\left(1-\frac{\varrho}{2}\right) \cdot S P$.

Proof The conjecture of the mid-price return is given by:

$$
\begin{aligned}
\Delta \widetilde{M}_{t} & =\Delta M_{t}+\frac{1}{2} \Omega B S_{t}-\frac{1}{2} \Omega B S_{t-1} \\
& =\frac{1}{2}(\varrho-1) \Omega B S_{t-1}+\frac{1}{2} \Omega B S_{t}+\frac{1}{2} \varrho(S P-\Omega) B S_{t-1}+\epsilon_{t}
\end{aligned}
$$

Then the covariance between two adjacent conjectures of mid-price returns is (the detail of the deduction is presented in appendix B and C.2):

$$
\begin{aligned}
& \operatorname{Cov}\left(\Delta \tilde{M}_{t}, \Delta \tilde{M}_{t-1}\right) \\
= & E\left(\Delta \widetilde{M}_{t} \cdot \Delta \widetilde{M}_{t-1}\right) \\
= & E\left(\epsilon_{t} \cdot \epsilon_{t-1}\right)+\Pi_{1} \cdot \Omega^{2}+\varrho \Pi_{3} \cdot(S P-\Omega) \cdot \Omega \\
& +\frac{1}{4} \varrho^{2}\left(B S_{t-1} \cdot B S_{t-2}\right) \cdot\left[(S P-\Omega)^{2}\right]
\end{aligned}
$$

where

$$
\begin{aligned}
& \Pi_{1}=\frac{1}{4} E\left[(\varrho-1)^{2}\left(B S_{t-1} \cdot B S_{t-2}\right)+(\varrho-1) B S_{t} \cdot B S_{t-2}+(\varrho-1)+\left(B S_{t-1} \cdot B S_{t-2}\right)\right] \\
& \Pi_{3}=\frac{1}{4} E\left[1+2(\varrho-1)\left(B S_{t-1} \cdot B S_{t-2}\right)+B S_{t} \cdot B S_{t-2}\right]
\end{aligned}
$$

Equation (27) suggests that when there are IC\&AS components, the covariance of the conjectural midprice returns $\left(\operatorname{Cov}\left(\Delta \widetilde{M}_{t}, \Delta \widetilde{M}_{t-1}\right)\right)$ is no longer a function of conjectural errors $(\Omega)$ only but also a function of the true spread $(S P)$.

Unlike the simple version of the estimator, the right hand side of Equation (B.8) is quadratic in $(S P-\Omega)$ and $\Omega$ instead of $\Omega$ only. The covariance of adjacent conjectural mid-price returns will not be maximised when $\Omega=0$. In other words, the estimator will be biased.

We now discuss possible errors if we still let $(S P-\Omega)$ which maximises the covariance between two adjacent conjectures of mid-price returns $\operatorname{Cov}\left(\Delta \widetilde{M}_{t}, \Delta \widetilde{M}_{t-1}\right)$ be the estimate of the true spread. Thus, the estimate is given by:

$$
\widehat{S P}=-\left[\frac{-2 \Pi_{1} S P+\Pi_{3} S P \varrho}{2\left(\Pi_{1}+\frac{1}{4} E\left(B S_{t-1} \cdot B S_{t-2}\right) \varrho^{2}-\Pi_{3} \varrho\right)}\right]
$$

where $\widehat{S P}$ is the value of $(S P-\Omega)$ which maximises $\operatorname{Cov}\left(\Delta \widetilde{M}_{t}, \Delta \widetilde{M}_{t-1}\right)$, and is the estimate of the true spread.

When there are IC\&AS components of the spread. The estimated spread should be (the detail of the deduction is presented in appendix C.2):

$$
\widehat{S P}=\left(1-\frac{\varrho}{2}\right) \cdot S P
$$

Q.E.D. 
Equation (30) suggests that when the transaction cost is not the only component of the spread, the estimator will underestimate the true spread. In the simulation section, an adjustment will be introduced to overcome this issue.

When there are no IC\&AS components of the spread $(\varrho=0)$ and no autocorrelated order flows $\left(E\left(B S_{t-1} \cdot B S_{t-2}\right)=0\right.$ and $\Pi_{1}=-1$ and $\left.\Pi_{3}=1\right)$, the equation above becomes:

$$
\widehat{S P}=S P
$$

and thus,

$$
\Omega=0
$$

The equations above suggest that under the ideal conditions, Equation (29) reduces to the simple version of the estimator and in this circumstance, the estimator is unbiased.

It can be shown that the estimator will not be influenced by the autocorrelation of order flows.

\section{Simulation Experiments}

In this section, simulated data are used to examine the performance of the new estimator. The aim of this section is to assess the effects of two factors on the performance of the estimators: mid-price changes caused by order flows (sometimes known as price impact) and current and lagged feedback trading. The performance of the Roll, Huang and Stoll and Corwin and Schultz models are also presented for comparison, for the reasons discussed below.

Roll (1984) establishes a spread estimation model based on the negative serial correlation of returns induced by the spread when trades switch direction. The Roll model considers the order-processing cost only and requires only transactions prices and not trade direction, which is frequently not known. The spread is estimated from the auto-covariance of price returns. The limitations of the original Roll estimator are addressed in the next generation of Roll estimators such as Glosten and Harris (1988) (adverse selection cost), Choi et al. (1988) (autocorrelation of order flows), Stoll (1989) and George et al. (1991) (price impact of order flows). These estimators either require additional information which is generally not available (for example, autocorrelation of mid-prices) or are incorporated into the HS model, so we do not consider these estimators in the comparison.

The Huang-Stoll model is an obvious comparator since it is the standard model to use when trade direction is known. The Corwin-Schultz estimator is used since it appears to be the best of several low-frequency estimators. Goyenko et al. (2009) and Corwin and Schultz (2012) show that the CorwinSchultz estimator is better than the Lesmond et al. (1999) estimator. The Holden (2009) low-frequency estimator cannot be evaluated by simulation experiments.

There are 500 replications simulated for each case. There are 432000 periods in a replication. Let one period represent one minute, and there is one trade per minute. Thus there are 300 trading days (1440 minutes and 1440 trades per day). For each replication, data are considered in various sampling periods: tick-by-tick, five minutes, fifteen minutes, one hour, four hours, twelve hours and 24 hours. Thus, there are eight subgroups for each replication. For five-minute intervals, only every fifth trade is used and the intervening trades discarded, and similarly for longer intervals.

Each replication includes data on order flows, bid-ask spreads, mid-prices, and translation prices. Data are generated according to the following system. An order has two possible values 1 and -1 . Order flows are either random or positively correlated with current and (possibly) lagged mid-price returns (the feedback-trading case). Formally, the order flow series is given by

$$
\begin{aligned}
& B S_{t}=\psi F\left(\Delta M_{t}+\eta \Delta M_{t-1}\right)+(1-\psi) \omega_{t} \\
& \psi=0 \text { or } 1
\end{aligned}
$$

where $B S_{t}$ is the order flow, which is either random $(\psi=0)$ or a function $F\left(\Delta M_{t-1}+\eta \Delta M_{t}\right)$ is a function of the past mid-price returns $(\psi=1)$, which suggests the existence of feedback trading. $\eta$ describes the existence of lagged feedback trading. For example, $\eta=0.5$ suggests that lagged feedback trading is $50 \%$ weaker than current feedback trading. $\omega_{t}$ is a binomial random variable, which follows a binomial distribution with one trial and $50 \%$ probability i.e. $B(1,0.5)$. It suggests that order flows are drawn from a binomial distribution randomly and both the buy and sell orders carry the same weight in the series. The function $F(\cdot)$ reflects the following relationship between order flows and past mid-price returns.

$$
B S_{t} \sim \begin{cases}B(1, \kappa) & \text { if } \Delta M_{t}>0 \\ B(1,1-\kappa) & \text { if } \Delta M_{t}<0\end{cases}
$$


where $B(1, \kappa)$ is a binomial distribution with one trial and $\kappa$ probability. When $\kappa=0.5$, there is no feedback trading, and when $\kappa>0.5$, there is positive feedback trading and vice versa.

Mid-price returns are generated using the following equation,

$$
\Delta M_{t}=\varrho B S_{t-1} \cdot \frac{S P_{t}}{2}+\epsilon_{t}
$$

where $\epsilon_{t}$ follows a normal distribution with zero mean and standard deviation $\sigma ; S P_{t}$ is the bid-ask spread which is assumed fixed. $\varrho$ is the fraction of the spread that is caused by inventory control and asymmetric information, and thus $(1-\varrho)$ represents the order-processing part of the spread. When $\varrho=0$, the order-processing part is the only component of the spread, and mid-price follow a random walk process.

Transaction prices are generated by

$$
s_{t}=M_{t}+\frac{S P_{t}}{2} \cdot B S_{t}
$$

where $s_{t}$ is the transaction price.

\subsection{Ideal Conditions}

In this section, the ideal case for the estimators is considered, where order flows are random, mid-prices follow a random walk process and the spread is fixed. Under these circumstances, the new estimator is unbiased. Formally, the standard deviations of mid-price returns is $\sigma=0.0002$, which is similar to that observed for major currencies in foreign exchange markets ${ }^{\mathrm{a}}$. Let $\psi=0$ in Equation (33), which suggests that order flows are random. Let $\varrho=0$ in Equation (35), which suggests that the mid-price follows a random walk process and the spread is fixed at 0.0003 . The system is given by,

$$
\begin{aligned}
& B S_{t}=\omega_{t} \\
& \omega_{t} \sim B(1,0.5) \\
& \Delta M_{t}=\epsilon_{t} \\
& \epsilon_{t} \sim N\left(0,4 \times 10^{-8}\right) \\
& S P_{t}=0.0003 \\
& s_{t}=M_{t}+\frac{S P_{t}}{2} \cdot B S_{t}
\end{aligned}
$$

Five hundred replications, each of which has 432000 periods, are generated according to the system above. Each replication has eight subgroups according to various sampling periods.

Transaction returns and order flows are used for estimations. The standard deviation of mid-price returns is also calculated. Thus, for every subgroup, there are 500 estimated spreads for each estimator and 500 standard deviations of mid-price returns.

The results are presented in Table 1. The first column shows the results when every transaction is used (tick-by-tick data). The other columns show the results when the transactions are sampled at increasingly long intervals, from five minutes to 24 hours. There are four panels which report the summary statistics and the results of the estimators respectively. The rows in each panel are as follows. Midstd reports the average of the standard deviations of mid- price returns over the relevant interval. Estimates indicates the average of estimated spreads, and Relative Estimates shows the ratio of this to the true spread. Est-Std reports the standard deviations of the estimated spreads. RMSE is the root mean square error, or the standard deviation of the estimates about the true spread, so it incorporates the effect of bias as well as the standard deviation of the estimates about their own mean. It is the best indicator of the likely error in an estimate of the spread from an individual series.

The row of Midstd shows the time interval and the standard deviation of mid-price returns have a positive relationship, as a result of the random walk in returns. In the tick-by-tick case, the average standard deviation of mid-price returns is $2 \times 10^{-4}$ which is the same as the setting of the system. In the 24-hour case, the standard deviation is $7.58 \times 10^{-3}$. Thus the ratio of the spread to the standard deviation varies from 1.5 in the tick-by-tick case to 0.0396 at 24 hours. Spreads are harder to estimate when this ratio is smaller, and hence the standard deviation and RMSE increase with the time interval.

\footnotetext{
a A referee suggests that we also run simulations calibrated to the stock market. If we set the ratio of the spread to the standard deviation of daily returns to one (i.e. the spread is much larger than in Table 1), as is typical of the stock market, the relative performance of estimators is similar to that shown in Table 1 . The results of these simulations can be found in the online appendix.
} 
It can be seen from Table 1 that both the new estimator and the HS estimator are highly accurate in tick-by-tick data, with an RMSE of less than $0.25 \%$ of the true spread of 0.0003 . As the sampling frequency falls, the RMSE rises quickly, exceeding $12 \%$ of the true spread in one-hour samples. The performance of the two estimators is extremely similar.

Table 1 Ideal Conditions

\begin{tabular}{|c|c|c|c|c|c|c|c|c|}
\hline & Tick & 5-Min & 15-Min & 30-Min & 1-Hour & 4-Hour & 12-Hour & 24-Hour \\
\hline Midstd $\times 10^{-2}$ & 0.0200 & 0.0447 & 0.0775 & 0.110 & 0.155 & 0.310 & 0.536 & 0.758 \\
\hline \multicolumn{9}{|c|}{ The New Estimator } \\
\hline Estimates $\times 10^{-3}$ & 0.300 & 0.300 & 0.300 & 0.300 & 0.298 & 0.310 & 0.289 & 0.278 \\
\hline Relative Estimates & 1 & 1 & 1 & 1 & 0.993 & 1.033 & 0.963 & 0.927 \\
\hline Est-Std $\times 10^{-3}$ & 0.000658 & 0.00302 & 0.00884 & 0.0177 & 0.0375 & 0.123 & 0.423 & 0.850 \\
\hline RMSE $\times 10^{-3}$ & 0.000658 & 0.00302 & 0.00884 & 0.0177 & 0.0376 & 0.123 & 0.423 & 0.850 \\
\hline \multicolumn{9}{|c|}{ Huang and Stoll 1997} \\
\hline Estimates $\times 10^{-3}$ & 0.300 & 0.300 & 0.301 & 0.301 & 0.301 & 0.301 & 0.282 & 0.280 \\
\hline Relative Estimates & 1 & 1 & 1.003 & 1.003 & 1.003 & 1.003 & 0.94 & 0.933 \\
\hline Est-Std $\times 10^{-3}$ & 0.000622 & 0.00289 & 0.00898 & 0.0169 & 0.0371 & 0.146 & 0.414 & 0.865 \\
\hline RMSE $\times 10^{-3}$ & 0.000622 & 0.00289 & 0.00904 & 0.0169 & 0.0371 & 0.146 & 0.414 & 0.865 \\
\hline \multicolumn{9}{|c|}{ Roll 1984} \\
\hline Estimates $\times 10^{-3}$ & 0.300 & 0.300 & 0.299 & 0.296 & 0.276 & 0.401 & 0.927 & 1.49 \\
\hline Relative Estimates & 1 & 1 & 0.997 & 0.987 & 0.920 & 1.337 & 3.090 & 4.967 \\
\hline Est-Std $\times 10^{-3}$ & 0.000853 & 0.00570 & 0.0246 & 0.0725 & 0.184 & 0.463 & 1.04 & 1.75 \\
\hline RMSE $\times 10^{-3}$ & 0.001 & 0.006 & 0.025 & 0.073 & 0.186 & 0.474 & 1.214 & 2.116 \\
\hline \multicolumn{9}{|c|}{ Corwin and Schultz 2012} \\
\hline Estimates $\times 10^{-3}$ & & & -0.0479 & 0.00988 & 0.075 & 0.257 & 0.489 & 0.728 \\
\hline Relative Estimates & & & -0.160 & 0.033 & 0.250 & 0.857 & 1.630 & 2.427 \\
\hline Est-Std $\times 10^{-3}$ & & & 0.00449 & 0.00803 & 0.0169 & 0.0667 & 0.215 & 0.399 \\
\hline RMSE $\times 10^{-3}$ & & & 0.348 & 0.290 & 0.226 & 0.079 & 0.286 & 0.585 \\
\hline
\end{tabular}

There are 500 replications. There are 432000 periods, each of which represents one minute, in each replication. Data of each replication are generated according to the following system. The order flow is drawn from a binomial distribution, i.e. $B S_{t} \sim B(1,0.5)$. The mid-price return is drawn from a normal distribution of which the mean is zero and the variance is $4 \times 10^{-8}$, i.e. $\Delta M_{t} \sim N\left(0,4 \times 10^{-8}\right)$. The spread is fixed and equals to 0.0003 , i.e. $S P_{t}=0.0003$. The transaction price is the mid-price plus or minus a half spread, i.e. $s_{t}=M_{t}+\frac{S P_{t}}{2} \cdot B S_{t}$. Each replication is also sampled into longer time intervals: five-minute, fifteen-minute, thirty-minute, one-hour, four-hour, twelve-hour and twenty-four- hour, and only the close observations are kept. Thus, there are eight subgroups for each replication. For each subgroup, the standard deviation of mid-price returns, and the estimated spread are collected. Midstd is the average of the standard deviations of mid-price returns.

Estimates is the average of the estimated spreads.

Relative Estimate represents the average of estimated spreads divided by the true spread. It is one if the estimate equals the true spread.

Est-Std is the standard deviation of the estimated spreads.

$R M S E$ is the Root Mean Square Error. 


\subsection{One-Period Feedback Trading}

In this section, most settings are the same as the ones in section (4.1) except that now order flows are assumed to be influenced by the latest mid-price returns. Thus all the differences of the performance of the estimators can be imputed to the existence of feedback trading. Let $\psi=1$ and $\eta=0$, which suggests that there is only current feedback trading. Under these circumstances, all the estimators are biased. However, the new estimator should have the smallest error and the HS estimator should have the greatest error. Formally, let $\psi=1$ and $\eta=0$ in Equation (33), which suggests that order flows affected by the past period mid-price returns. Let $\kappa=0.65^{\mathrm{b}}$, which implies that there is positive net feedback trading. As in the previous case, the spread is fixed at 0.0003 . The system is given by,

$$
\begin{aligned}
& B S_{t} \sim \begin{cases}B(1,0.65) & \text { if } \Delta M_{t}>0 \\
B(1,0.35) & \text { if } \Delta M_{t}<0\end{cases} \\
& \Delta M_{t}=\epsilon_{t} \\
& \epsilon_{t} \sim N\left(0,9 \times 10^{-12}\right) \\
& S P_{t}=0.0003 \\
& s_{t}=M_{t}+\frac{S P_{t}}{2} \cdot B S_{t}
\end{aligned}
$$

The results are shown in Table 2. The bias in the estimators can be seen in the relative estimate for the tick-by-tick case (a bias of $+16 \%$ for the new estimator, and $+32 \%$ for the HS estimator). Since the standard deviation of the estimates is very small at short time intervals, the RMSE is dominated by the bias in these cases (up to 30-minute intervals). The bias is slightly larger at longer time intervals for both estimators. Clearly, however, the new estimator outperforms the HS estimator in the presence of feedback trading, as predicted by our earlier analysis.

\footnotetext{
$\mathrm{b}$ We have also done some simulations with a higher value of . For $\kappa=0.85$, the biases are larger in every case than those shown in Table 2, but the relative performance of the different estimators is similar. The results of these simulations can be found in the online appendix.
} 
Table 2 One-period Feedback Trading

\begin{tabular}{|c|c|c|c|c|c|c|c|c|}
\hline Mid-price returns SD $\times 10^{-3}$ & $\begin{array}{c}\text { Tick } \\
0.200\end{array}$ & $\begin{array}{c}\text { 5-Min } \\
0.447\end{array}$ & $\begin{array}{c}\text { 15-Min } \\
0.774\end{array}$ & $\frac{30-\mathrm{Min}}{1.09}$ & $\frac{1 \text {-Hour }}{1.55}$ & $\frac{4 \text {-Hour }}{3.09}$ & $\frac{12 \text {-Hour }}{5.36}$ & $\begin{array}{c}\text { 24-Hour } \\
7.58\end{array}$ \\
\hline Spread/(returns SD) & 1.5 & 0.671 & 0.387 & 0.273 & 0.194 & 0.0968 & 0.0560 & 0.0396 \\
\hline $\operatorname{Cov}\left(\Delta M_{t}, B S_{t}\right) \times 10^{-3}$ & 0.0479 & 0.0479 & 0.0479 & 0.0480 & 0.0477 & 0.0447 & 0.0584 & 0.0544 \\
\hline \multicolumn{9}{|c|}{ The New Estimator } \\
\hline Relative Estimate & 1.16 & 1.16 & 1.16 & 1.15 & 1.17 & 1.16 & 1.20 & 1.29 \\
\hline Est-Std $\times 10^{-3}$ & 0.000683 & 0.00305 & 0.00923 & 0.0180 & 0.0373 & 0.139 & 0.439 & 0.879 \\
\hline RMSE $\times 10^{-3}$ & 0.0480 & 0.0481 & 0.0489 & 0.0494 & 0.0624 & 0.147 & 0.443 & 0.883 \\
\hline \multicolumn{9}{|c|}{ Huang and Stoll 1997} \\
\hline Relative Estimate & 1.32 & 1.32 & 1.32 & 1.32 & 1.33 & 1.33 & 1.36 & 1.41 \\
\hline Est-Std $\times 10^{-3}$ & 0.000579 & 0.00304 & 0.00889 & 0.0175 & 0.0369 & 0.145 & 0.446 & 0.876 \\
\hline RMSE $\times 10^{-3}$ & 0.0960 & 0.0960 & 0.0954 & 0.0966 & 0.105 & 0.176 & 0.459 & 0.884 \\
\hline \multicolumn{9}{|c|}{ Roll 1984} \\
\hline Relative Estimates & 1.150 & 1.150 & 1.150 & 1.117 & 1.043 & 1.533 & 3.073 & 5.267 \\
\hline Est-Std $\times 10^{-3}$ & 0.000923 & 0.00528 & 0.0218 & 0.0669 & 0.177 & 0.497 & 1.06 & 1.79 \\
\hline RMSE $\times 10^{-3}$ & 0.045 & 0.045 & 0.050 & 0.076 & 0.177 & 0.522 & 1.229 & 2.201 \\
\hline \multicolumn{9}{|c|}{ Corwin and Schultz 2012} \\
\hline Relative Estimates & & & -0.045 & 0.153 & 0.373 & 1.000 & 1.827 & 2.613 \\
\hline Est-Std $\times 10^{-3}$ & & & 0.00439 & 0.00885 & 0.0169 & 0.0694 & 0.208 & 0.388 \\
\hline RMSE $\times 10^{-3}$ & & & 0.313 & 0.254 & 0.189 & 0.069 & 0.324 & 0.620 \\
\hline
\end{tabular}

There are 500 replications. There are 432000 periods, each of which represents one minute, in each replication. Data of each replication are generated according to the following system. The order flow is positively autocorrelated. The mid-price return is drawn from a normal distribution of which the mean is zero and the variance is $4 \times 10^{-8}$, i.e. $\Delta M_{t} \sim N\left(0,4 \times 10^{-8}\right)$. Order flows is positively correlated to mid-price returns. The probability of a buy (sell) order being after a positive (negative) return is $65 \%$. i.e. The spread is fixed and equals to 0.0003 , i.e. $B S_{t} \sim B(1,0.65)$ if $\Delta M_{t}>0$ and $B S_{t} \sim B(1,0.35)$ if $\Delta M_{t}<0$. $S P_{t}=0.0003$. The transaction price is the mid-price plus or minus a half spread, i.e. $s_{t}=M_{t}+\frac{S P_{t}}{2} \cdot B S_{t}$. Each replication is also sampled into longer time intervals: five-minute, fifteen-minute, thirty-minute, one-hour, four-hour, twelve-hour and twenty-four- hour, and only the close observations are kept. Thus, there are eight subgroups for each replication. For each subgroup, the standard deviation of mid-price returns, and the estimated spread are collected.

$\operatorname{Cov}\left(\Delta M_{t}, B S_{t}\right)$ is the covariance of mid-price returns and order flows, which reflects the existence of feedback trading. The other settings are the same as Table (1) 


\subsection{Two-Period Feedback Trading}

In this section, most settings are the same as the ones in section (4.2) except that now e add lagged feedback trading. Let $\psi=1$ and $\eta=0.5$ which implies that there is both current and lagged feedback trading, with lagged feedback trading in the same direction as but $50 \%$ weaker than current trading ${ }^{\mathrm{c}}$. Net feedback trading is the summation of them: $\Delta M_{t}+0.5 \Delta M_{t-1}$. In this section, order flows are random; order flows are influenced by the past mid-price returns; and the spread is fixed. Under these circumstances, all the estimators are biased. However, the new estimator should have the least error and the HS estimator should have the greatest error. Formally, let $\psi=1$ and $\eta=0.5$ in Equation (33), which suggests that order flows affected by past two periods mid-price returns ${ }^{\mathrm{d}}$. Let $\kappa=0.65$, which suggest there is positive net feedback trading. The spread is still fixed at 0.0003 . The system is given by,

$$
\begin{aligned}
& B S_{t} \sim \begin{cases}B(1,0.65) & \text { if } \Delta M_{t}+0.5 \Delta M_{t-1}>0 \\
B(1,0.35) & \text { if } \Delta M_{t}+0.5 \Delta M_{t-1}<0\end{cases} \\
& \Delta M_{t}=\epsilon_{t} \\
& \epsilon_{t} \sim N\left(0,9 \times 10^{-12}\right) \\
& S P_{t}=0.0003 \\
& s_{t}=M_{t}+\frac{S P_{t}}{2} \cdot B S_{t}
\end{aligned}
$$

The results are presented in Table 3. The HS estimator performs slightly better than it did in Table 2 (current feedback trading only) in the tick-by-tick case, with a bias of $+28.7 \%$ compared with $+32 \%$ in Table 2. However it performs worse than in Table 2 at any longer time interval (for example at five minutes the bias for the HS estimator rises to $+42.7 \%$, compared with $+32 \%$ in Table 2 ). The new estimator, by contrast, performs even better than in Table 2 in the tick-by-tick case, because of the offsetting effect of current and lagged feedback trading shown in Equation (19). The bias of the new estimator is only $+7.3 \%$ in the tick-by-tick case, compared with $+16 \%$ in Table 2 . At longer time intervals the new estimator, like the HS estimator, performs worse in Table 3 than in Table 2, but its bias is substantially less at all time intervals than that of the HS estimator.

\footnotetext{
c We have also carried out some simulations with lower values of $\eta$. The results are intermediate between those of Tables 2 $(\eta=0)$ and $3(\eta=0.5)$. The results of these simulations can be found in the online appendix.

$\mathrm{d}$ One referee points out that by introducing lagged feedback trading, trade direction indicators will also exhibit autocorrelation. Furthermore, as the referee notes, the new estimator is not influenced by autocorrelated trade direction indicators. Bleaney and Li (2015) show that the HS and the CS estimators are not influenced by trade direction indicators, and the Roll estimator is influenced by them in the tick-by-tick case.
} 
Table 3 Two-Period Feedback Trading

\begin{tabular}{|c|c|c|c|c|c|c|c|c|}
\hline & Tick & 5-Min & 15-Min & 30-Min & 1-Hour & 4-Hour & 12-Hour & 24-Hour \\
\hline Midstd $\times 10^{-3}$ & 0.200 & 0.514 & 0.815 & 1.12 & 1.57 & 3.11 & 5.36 & 7.58 \\
\hline $\operatorname{Cov}\left(\Delta M_{t}, B S_{t}\right) \times 10^{-4}$ & 0.428 & 0.428 & 0.428 & 0.429 & 0.430 & 0.427 & 0.427 & 0.430 \\
\hline $\operatorname{Cov}\left(\Delta M_{t-1}, B S_{t}\right) \times 10^{-5}$ & & 0.00421 & -0.00239 & -0.00424 & -0.00943 & 0.0472 & -0.0322 & -0.0317 \\
\hline \multicolumn{9}{|c|}{ The New Estimator } \\
\hline Estimates $\times 10^{-3}$ & 0.322 & 0.364 & 0.365 & 0.365 & 0.362 & 0.364 & 0.372 & 0.358 \\
\hline Relative Estimates & 1.073 & 1.213 & 1.217 & 1.217 & 1.207 & 1.213 & 1.24 & 1.193 \\
\hline Est-Std $\times 10^{-3}$ & 0.000592 & 0.00291 & 0.00948 & 0.0193 & 0.0359 & 0.145 & 0.460 & 0.896 \\
\hline RMSE $\times 10^{-3}$ & 0.0220 & 0.0641 & 0.0657 & 0.0678 & 0.0716 & 0.158 & 0.466 & 0.898 \\
\hline \multicolumn{9}{|c|}{ Huang and Stoll 1997} \\
\hline Estimates $\times 10^{-3}$ & 0.386 & 0.428 & 0.429 & 0.429 & 0.428 & 0.434 & 0.456 & 0.451 \\
\hline Relative Estimates & 1.287 & 1.427 & 1.43 & 1.43 & 1.427 & 1.447 & 1.52 & 1.503 \\
\hline Est-Std $\times 10^{-3}$ & 0.000556 & 0.00299 & 0.00868 & 0.0180 & 0.0348 & 0.150 & 0.462 & 0.924 \\
\hline RMSE $\times 10^{-3}$ & 0.0860 & 0.128 & 0.129 & 0.130 & 0.133 & 0.201 & 0.488 & 0.936 \\
\hline \multicolumn{9}{|c|}{ Roll 1984} \\
\hline Estimates $\times 10^{-3}$ & 0.314 & 0.358 & 0.361 & 0.351 & 0.323 & 0.475 & 0.862 & 1.37 \\
\hline Relative Estimates & 1.047 & 1.193 & 1.203 & 1.170 & 1.077 & 1.583 & 2.873 & 4.567 \\
\hline Est-Std $\times 10^{-3}$ & 0.000982 & 0.00507 & 0.0201 & 0.0646 & 0.176 & 0.471 & 1.06 & 1.74 \\
\hline RMSE $\times 10^{-3}$ & 0.014 & 0.058 & 0.064 & 0.082 & 0.177 & 0.502 & 1.200 & 2.043 \\
\hline \multicolumn{9}{|c|}{ Corwin and Schultz 2012} \\
\hline Estimates $\times 10^{-3}$ & & & -0.0116 & 0.0485 & 0.115 & 0.301 & 0.552 & 0.774 \\
\hline Relative Estimates & & & -0.0387 & 0.162 & 0.383 & 1.003 & 1.84 & 2.58 \\
\hline Est-Std $\times 10^{-3}$ & & & 0.00452 & 0.00791 & 0.0168 & 0.0685 & 0.202 & 0.383 \\
\hline RMSE $\times 10^{-3}$ & & & 0.312 & 0.252 & 0.186 & 0.0685 & 0.323 & 0.609 \\
\hline
\end{tabular}

There are 500 replications. There are 432000 periods, each of which represents one minute, in each replication. Data of each replication are generated according to the following system. The order flow is positively autocorrelated. The mid-price return is drawn from a normal distribution of which the mean is zero and the variance is $4 \times 10^{-8}$, i.e. $\Delta M_{t} \sim N\left(0,4 \times 10^{-8}\right)$. Order flows is positively correlated to past mid-price returns. The probability of a buy (sell) order with positive (negative) net feedback trading is $65 \%$. i.e. The spread is fixed and equals to 0.0003 , i.e. $B S_{t} \sim B(1,0.65)$ if $\Delta M_{t}+0.5 \Delta M_{t-1}>0$ and $B S_{t} \sim B(1,0.35)$ if $\Delta M_{t}+0.5 \Delta M_{t-1}<0 . S P_{t}=0.0003$. The transaction price is the mid-price plus or minus a half spread, i.e. $s_{t}=M_{t}+\frac{S P_{t}}{2} \cdot B S_{t}$. Each replication is also sampled into longer time intervals: five-minute, fifteen-minute, thirty-minute, one-hour, four-hour, twelve-hour and twenty-four- hour, and only the close observations are kept. Thus, there are eight subgroups for each replication. For each subgroup, the standard deviation of mid-price returns, and the estimated spread are collected.

Midstd is the average of the standard deviations of mid-price returns.

$\operatorname{Cov}\left(\Delta M_{t}, B S_{t}\right)$ is the covariance of mid-price returns and order flows, which reflects the existence of current feedback trading.

$\operatorname{Cov}\left(\Delta M_{t-1}, B S_{t}\right)$ is the covariance of mid-price returns and order flows, which reflects the existence of lagged feedback trading.

The other settings are the same as Table (1) 


\subsection{Inventory Control and Asymmetric Information Components}

In this section, most settings are the same as the ones in section (4.1) except that the mid-price return is now assumed to be influenced by the past order flow, and thus there are inventory control and the asymmetric information components to the spread. Order flow is assumed to be random, so there is no feedback trading. Let $\varrho=\frac{1}{3}$, which suggests that the inventory control and asymmetric information parts contribute one third of the total spread. Under these circumstances, the new estimator is biased, but the HS estimator is unbiased. Formally, let $\psi=0$ in Equation (33), which suggests that order flows are random. The spread is 0.0003 , as before. The system is given by:

$$
\begin{aligned}
& B S_{t}=\omega_{t} \\
& \omega_{t} \sim B(1,0.5) \\
& \Delta M_{t}=\frac{1}{3} B S_{t-1} \cdot \frac{S P_{t}}{2}+\epsilon_{t} \\
& \Delta M_{t}=\frac{2}{3} B S_{t-1} \cdot \frac{S P_{t}}{2}+\epsilon_{t} \\
& \epsilon_{t} \sim N\left(0,4 \times 10^{-8}\right) \\
& S P_{t}=0.0003 \\
& s_{t}=M_{t}+\frac{S P_{t}}{2} \cdot B S_{t}
\end{aligned}
$$

The results are presented in Table (4), in which the second row ( $\varrho$ ) reports the coefficient of Equation (35) and represents the proportion of the IC \& AS components of the spread.

The standard deviation of mid-price returns is slightly greater than in the previous cases $\left(2.06 \times 10^{-4}\right.$ for tick-by-tick data, rising to $7.80 \times 10^{-3}$ for 24 -hour intervals). Thus the ratio of the spread to the standard deviation ranges from 1.46 to 0.0385 . The estimated $\varrho$ is close to $\frac{1}{3}$, which is the same as the setting, when the time interval is short. When the time interval is longer than one hour, $\varrho$ becomes unstable, because in relatively long runs the microstructure effects are weaker. While the HS estimator remains accurate, the new estimator underestimates the spread by $16.7 \%$, or half of $\varrho$, as predicted in our earlier theoretical discussion. 
Table 4 Inventory Control and Asymmetric Information Components

\begin{tabular}{|c|c|c|c|c|c|c|c|c|}
\hline & Tick & 5-Min & 15-Min & 30-Min & 1-Hour & 4-Hour & 12-Hour & 24-Hour \\
\hline Midstd $\times 10^{-2}$ & $\begin{array}{c}0.0206 \\
0.333 \\
\end{array}$ & $\begin{array}{c}0.0461 \\
0.333 \\
\end{array}$ & $\begin{array}{c}0.0798 \\
0.333\end{array}$ & $\begin{array}{l}0.113 \\
0.333 \\
\end{array}$ & $\begin{array}{l}0.160 \\
0.328\end{array}$ & $\begin{array}{l}0.319 \\
0.312 \\
\end{array}$ & $\begin{array}{l}0.552 \\
0.247\end{array}$ & $\begin{array}{l}0.780 \\
0.200\end{array}$ \\
\hline \multicolumn{9}{|c|}{ The New Estimator } \\
\hline Relative Estimates & 0.833 & 0.833 & 0.833 & 0.833 & 0.84 & 0.847 & 0.847 & 1.157 \\
\hline Est-Std $\times 10^{-3}$ & 0.000656 & 0.00305 & 0.00886 & 0.0182 & 0.0374 & 0.153 & 0.457 & 0.917 \\
\hline RMSE $\times 10^{-3}$ & 0.0500 & 0.0501 & 0.0508 & 0.0532 & 0.0609 & 0.160 & 0.459 & 0.918 \\
\hline \multicolumn{9}{|c|}{ Huang and Stoll 1997} \\
\hline Relative Estimates & 1 & 1 & 1 & 1 & 1.01 & 1.007 & 1.037 & 1.167 \\
\hline Est-Std $\times 10^{-3}$ & 0.000626 & 0.00312 & 0.00862 & 0.0173 & 0.0391 & 0.155 & 0.441 & 0.927 \\
\hline $\mathrm{RMSE} \times 10^{-3}$ & 0.000626 & 0.00312 & 0.00862 & 0.0173 & 0.0392 & 0.155 & 0.441 & 0.928 \\
\hline \multicolumn{9}{|c|}{ Roll 1984} \\
\hline Relative Estimates & 0.817 & 0.817 & 0.813 & 0.760 & 0.757 & 1.513 & 3.213 & 5.133 \\
\hline Est-Std $\times 10^{-3}$ & 0.000892 & 0.00683 & 0.0331 & 0.101 & 0.186 & 0.499 & 1.08 & 1.79 \\
\hline RMSE $\times 10^{-3}$ & 0.055 & 0.055 & 0.065 & 0.124 & 0.200 & 0.522 & 1.268 & 2.178 \\
\hline \multicolumn{9}{|c|}{ Corwin and Schultz 2012} \\
\hline Relative Estimates & & & -0.304 & -0.120 & 0.095 & 0.730 & 1.590 & 2.387 \\
\hline Est-Std $\times 10^{-3}$ & & & 0.00442 & 0.00883 & 0.0167 & 0.0692 & 0.204 & 0.431 \\
\hline RMSE $\times 10^{-3}$ & & & 0.391 & 0.336 & 0.272 & 0.107 & 0.270 & 0.599 \\
\hline
\end{tabular}

There are 500 replications. There are 432000 periods, each of which represents one minute, in each replication. Data of each replication are generated according to the following system. The order flow is drawn from a binomial distribution, i.e. $B S_{t} \sim B(1,0.5)$. The mid-price return is influenced by the past order flow and a random shock drawn from a normal distribution of which the mean is zero and the variance is $4 \times 10^{-8}$. Thus there are the inventory control and the asymmetric information components in the spread. Formally, the mid-price returns are given by, $\Delta M_{t}=\frac{1}{3} B S_{t-1} \cdot \frac{S P_{t}}{2}+\varepsilon_{t}$ where $\varepsilon_{t} \sim N\left(0,4 \times 10^{-8}\right)$. The spread is fixed and equals to 0.0003 , i.e. $S P_{t}=0.0003$. The transaction price is the mid-price plus or minus a half spread, i.e. $s_{t}=M_{t}+\frac{S P_{t}}{2} \cdot B S_{t}$. Each replication is also sampled into longer time intervals: five-minute, fifteen-minute, thirty-minute, one-hour, four-hour, twelve-hour and twenty-four- hour, and only the close observations are kept. Thus, there are eight subgroups for each replication. For each subgroup, the standard deviation of mid-price returns, and the estimated spread are collected.

The other settings are the same as Table (1) 


\subsection{Both Feedback Trading and Price Impact}

In this section, we investigate the performance of the two estimators in the presence of both feedback trading (which favours the new estimator) and inventory control and asymmetric information components of the spread (which favours the HS estimator). We assume two-period feedback trading, as in section (4.3), and we investigate two separate settings for $\varrho$ : one-third, as in section (4.4), and a larger one of two-thirds. Thus $\psi=1, \eta=0.5$ and $\varrho=\frac{1}{3}$ or $\frac{2}{3}$. The system is given by:

$$
\begin{aligned}
& B S_{t} \sim \begin{cases}B(1,0.65) & \text { if } \Delta M_{t}+0.5 \Delta M_{t-1}>0 \\
B(1,0.35) & \text { if } \Delta M_{t}+0.5 \Delta M_{t-1}<0\end{cases} \\
& \Delta M_{t}=\frac{1}{3} B S_{t-1} \cdot \frac{S P_{t}}{2}+\epsilon_{t} \quad \text { in table } 5 \\
& \Delta M_{t}=\frac{2}{3} B S_{t-1} \cdot \frac{S P_{t}}{2}+\epsilon_{t} \quad \text { in table } 6 \\
& \epsilon_{t} \sim N\left(0,9 \times 10^{-12}\right) \\
& S P_{t}=0.0003 \\
& s_{t}=M_{t}+\frac{S P_{t}}{2} \cdot B S_{t}
\end{aligned}
$$

Table (5) shows the results for $\varrho=\frac{1}{3}$. As in the case of two-period feedback trading alone (Table 3), the HS estimator overestimates the spread considerably: by $28.7 \%$ in tick-by-tick data and by rather more in time-aggregated data. In fact the numbers for the HS estimator are very similar to those in Table (3); the price impact makes virtually no difference. For the new estimator the picture is very different. The underestimation associated with price impact offsets the overestimation caused by feedback trading. In tick-by-tick data the new estimator underestimates by $9 \%$, but overestimates slightly in time-aggregated data (by about $5 \%$ up to four hours, and by quite a bit more at longer intervals).

When $\varrho=\frac{2}{3}$, the simulation results are as show in Table (6). The HS results are very close to those shown in Table (5). For the new estimator, the higher value of $\varrho$ reduces the estimates, as expected. In tick-by-tick data the new estimator now underestimates by $25.7 \%$, and by $12 \%$ in five-minute data and by $10 \%$ in four-hour data, only overestimating at longer intervals.

\section{Discussion}

Our new spread estimator, based on a trial-and-error procedure, was shown to perform almost as well as the Roll, HS and CS estimators in ideal conditions of no price impact or feedback trading (Table 1). A little-recognised weakness of the Roll and HS estimators is that they are prone to overestimate the spread in the presence of (positive) feedback trading. Our new estimator also overestimates the spread in the presence of feedback trading, but by considerably less than the HS estimator does. Although the Roll estimator is slightly better than our new estimator at high sampling frequencies (about $1 \%$ in terms of error), the Roll estimator is much worse than our new estimator at lower sampling frequencies. With only current feedback trading, the overestimation bias of the new estimator is only half that of the HS estimator in tick-by-tick data (Table 2). With lagged feedback trading as well, the bias in the new estimator is even smaller, both absolutely and relative to the HS estimator (Table 3).

In the presence of inventory control and asymmetric information components of the spread, the HS estimator remains unbiased, because these elements are built into the HS estimation procedure (Table 4). The new estimator and the Roll estimator, however, underestimate to the tune of half of $\varrho$, where $\varrho$ is the proportion of the spread attributable to price impact. When both feedback trading and price impact effects are present (Tables 5 and 6), the new estimator and the Roll estimator benefit from the offsetting effects of the tendency to overestimate in the feedback trading case and to underestimate in the price impact case. The new estimator and the Roll estimator therefore tend to outperform the HS estimator, which performs as poorly in this case as in the pure feedback trading case. Furthermore, the new estimator outperforms the Roll estimator at all sampling frequencies.

The CS estimator is not influenced by either feedback trading and the presence of inventory control and asymmetric information components of the spread, but its estimates are not consistent across sampling frequencies and are significantly biased. At low sampling frequencies, the CS estimator has the lowest RMSE because of its low standard deviation. However, as in Bleaney and Li (2015), if the sample size is large, the CS estimator is outperformed even at low frequencies by the other estimators, whose accuracy improves markedly with the size of the sample, whereas the accuracy of the CS estimator does not. 
Table 5 Inventory Control and Asymmetric Information Components and Two-Period Feedback Trading

\begin{tabular}{|c|c|c|c|c|c|c|c|c|}
\hline & Tick & 5-Min & 15-Min & 30-Min & 1-Hour & 4-Hour & 12-Hour & 24-Hour \\
\hline $\begin{array}{l}\text { Midstd } \times 10^{-2} \\
\varrho \\
\operatorname{Cov}\left(\Delta M_{t}, B S_{t}\right) \times 10^{-4} \\
\operatorname{Cov}\left(\Delta M_{t-1}, B S_{t}\right) \times 10^{-5}\end{array}$ & $\begin{array}{c}0.0206 \\
0.333 \\
0.440 \\
2.14 \\
\end{array}$ & $\begin{array}{c}0.0535 \\
0.341 \\
0.440 \\
-0.00369 \\
\end{array}$ & $\begin{array}{c}0.0881 \\
0.342 \\
0.440 \\
-0.00163 \\
\end{array}$ & $\begin{array}{c}0.123 \\
0.338 \\
0.439 \\
-0.00717\end{array}$ & $\begin{array}{c}0.173 \\
0.334 \\
0.440 \\
-0.0370 \\
\end{array}$ & $\begin{array}{c}0.344 \\
0.329 \\
0.443 \\
-0.0164 \\
\end{array}$ & $\begin{array}{c}0.594 \\
0.360 \\
0.447 \\
-0.0311 \\
\end{array}$ & $\begin{array}{c}0.839 \\
0.319 \\
0.444 \\
-0.00629 \\
\end{array}$ \\
\hline Estimates $\times 10^{-3}$ & 0.273 & $\begin{array}{l}\text { The I } \\
0.314\end{array}$ & $\frac{v \text { Estimat }}{0.314}$ & 0.315 & 0.317 & 0.316 & 0.359 & 0.443 \\
\hline Relative Estimates & 0.91 & 1.047 & 1.047 & 1.05 & 1.057 & 1.053 & 1.197 & 1.477 \\
\hline Est-Std $\times 10^{-3}$ & 0.000602 & 0.00343 & 0.00993 & 0.0197 & 0.04 & 0.161 & 0.332 & 0.426 \\
\hline RMSE $\times 10^{-3}$ & 0.0270 & $\frac{0.0144}{\text { Huan }}$ & 0.0172 & 0.0248 & 0.0435 & 0.162 & 0.337 & 0.449 \\
\hline Estimates $\times 10^{-3}$ & 0.386 & 0.431 & 0.431 & 0.431 & 0.432 & 0.432 & 0.430 & 0.413 \\
\hline Relative Estimates & 1.287 & 1.437 & 1.437 & 1.437 & 1.44 & 1.44 & 1.433 & 1.377 \\
\hline Est-Std $\times 10^{-3}$ & 0.000615 & 0.00329 & 0.0105 & 0.0197 & 0.0428 & 0.171 & 0.487 & 0.939 \\
\hline RMSE $\times 10^{-3}$ & 0.0860 & 0.131 & 0.131 & 0.132 & 0.139 & 0.216 & 0.504 & 0.946 \\
\hline \multicolumn{9}{|c|}{ Roll 1984} \\
\hline Relative Estimates & 0.823 & 0.950 & 0.940 & 0.890 & 0.880 & 1.643 & 3.433 & 5.567 \\
\hline Est-Std $\times 10^{-3}$ & 0.00103 & 0.00715 & 0.0342 & 0.105 & 0.199 & 0.524 & 1.160 & 1.930 \\
\hline RMSE $\times 10^{-3}$ & 0.053 & 0.017 & 0.039 & 0.110 & 0.202 & 0.558 & 1.371 & 2.367 \\
\hline \multicolumn{9}{|c|}{ Corwin and Schultz 2012} \\
\hline Relative Estimates & & & -0.294 & -0.087 & 0.148 & 0.837 & 1.743 & 2.597 \\
\hline Est-Std $\times 10^{-3}$ & & & 0.00517 & 0.00976 & 0.0184 & 0.0746 & 0.222 & 0.426 \\
\hline RMSE $\times 10^{-3}$ & & & 0.388 & 0.326 & 0.256 & 0.089 & 0.315 & 0.641 \\
\hline
\end{tabular}

There are 500 replications. There are 432000 periods, each of which represents one minute, in each replication. Data of each replication are generated according to the following system. The order flow is drawn from a binomial distribution, i.e. $B S_{t} \sim B(1,0.5)$. The mid-price return is influenced by the past order flow and a random shock drawn from a normal distribution of which the mean is zero and the variance is $4 \times 10^{-8}$. Thus there are the inventory control and the asymmetric information components in the spread. Formally, the mid-price returns are given by, $\Delta M_{t}=\frac{1}{3} B S_{t-1} \cdot \frac{S P_{t}}{2}+\varepsilon_{t}$ where $\varepsilon_{t} \sim N\left(0,4 \times 10^{-8}\right)$. The spread is fixed and equals to 0.0003 , i.e. $S P_{t}=0.0003$. The transaction price is the mid-price plus or minus a half spread, i.e. $s_{t}=M_{t}+\frac{S P_{t}}{2} \cdot B S_{t}$. Each replication is also sampled into longer time intervals: five-minute, fifteen-minute, thirty-minute, one-hour, four-hour, twelve-hour and twenty-four- hour, and only the close observations are kept. Thus, there are eight subgroups for each replication. For each subgroup, the standard deviation of mid-price returns, and the estimated spread are collected.

The other settings are the same as Table (1)

An interesting point is that the spread estimates produced by our new estimator, the HS estimator and the Roll estimator, all of which are unbiased under ideal conditions, are not highly correlated in individual simulations. This means that an average of all three, which is still unbiased, has a lower estimation error than does any individual estimator. There is therefore a strong case for using several estimators in high-frequency data.

\section{Conclusions}

We have proposed a new bid-ask spread estimator based on the principle that the covariance of successive mid-price returns tends to be maximised at the true value of the spread. A grid search or trialand-error procedure for maximising this covariance over alternative conjectures about the spread may therefore be used to estimate the true spread. The information requirements are the same as for Huang and Stoll's (1997) estimator: transaction prices and trade direction. Theoretically it was shown that the new estimator overestimates the spread in the presence of positive feedback trading (a rise in price making a buy order more likely), but by considerably less than the Huang-Stoll estimator. Price impact causes the new estimator to underestimate the spread, with a bias equal to half the proportion of the spread represented by price impact. Simulation results confirm the theoretical findings. Simulation results for the combination of feedback trading and price impact show that the bias effects identified in the separate cases are approximately additive. This means that the Huang-Stoll estimator performs as poorly in the combined case as in the pure feedback trading case, whereas the new estimator tends to 
Table 6 Inventory Control and Asymmetric Information Components and Two-Period Feedback Trading

\begin{tabular}{|c|c|c|c|c|c|c|c|c|}
\hline & Tick & 5-Min & 15-Min & 30-Min & 1-Hour & 4-Hour & 12-Hour & 24-Hour \\
\hline $\begin{array}{l}\text { Midstd } \times 10^{-2} \\
\varrho \\
\operatorname{Cov}\left(\Delta M_{t}, B S_{t}\right) \times 10^{-4} \\
\operatorname{Cov}\left(\Delta M_{t-1}, B S_{t}\right) \times 10^{-5}\end{array}$ & $\begin{array}{c}0.0224 \\
0.667 \\
0.452 \\
2.14 \\
\end{array}$ & $\begin{array}{c}0.0577 \\
0.682 \\
0.452 \\
-0.00296 \\
\end{array}$ & $\begin{array}{c}0.0983 \\
0.683 \\
0.453 \\
-0.00992 \\
\end{array}$ & $\begin{array}{c}0.138 \\
0.681 \\
0.453 \\
-0.0132 \\
\end{array}$ & $\begin{array}{c}0.195 \\
0.666 \\
0.453 \\
-0.00441 \\
\end{array}$ & $\begin{array}{c}0.390 \\
0.670 \\
0.457 \\
-0.0295 \\
\end{array}$ & $\begin{array}{c}0.675 \\
0.709 \\
0.461 \\
-0.0374 \\
\end{array}$ & $\begin{array}{c}0.954 \\
0.702 \\
0.46 \\
-0.0908 \\
\end{array}$ \\
\hline \multicolumn{9}{|c|}{ The New Estimator } \\
\hline Estimates $\times 10^{-3}$ & 0.223 & 0.264 & 0.264 & 0.264 & 0.269 & 0.270 & 0.356 & 0.422 \\
\hline Relative Estimates & 0.743 & 0.880 & 0.880 & 0.880 & 0.897 & 0.900 & 1.187 & 1.407 \\
\hline Est-Std $\times 10^{-3}$ & 0.00124 & 0.00425 & 0.0108 & 0.0232 & 0.0461 & 0.167 & 0.356 & 0.425 \\
\hline $\mathrm{RMSE} \times 10^{-3}$ & 0.077 & 0.036 & 0.038 & 0.043 & 0.056 & 0.170 & 0.360 & 0.442 \\
\hline \multicolumn{9}{|c|}{ Huang and Stoll 1997} \\
\hline Estimates $\times 10^{-3}$ & 0.386 & 0.433 & 0.433 & 0.433 & 0.434 & 0.448 & 0.465 & 0.409 \\
\hline Relative Estimates & 1.287 & 1.443 & 1.443 & 1.443 & 1.447 & 1.493 & 1.550 & 1.363 \\
\hline Est-Std $\times 10^{-3}$ & 0.000615 & 0.00329 & 0.0105 & 0.0197 & 0.0428 & 0.171 & 0.485 & 0.93 \\
\hline $\mathrm{RMSE} \times 10^{-3}$ & 0.086 & 0.133 & 0.133 & 0.135 & 0.143 & 0.242 & 0.582 & 1.105 \\
\hline \multicolumn{9}{|c|}{ Roll 1984} \\
\hline Estimates $\times 10^{-3}$ & 0.153 & 0.182 & 0.167 & 0.155 & 0.221 & 0.509 & 1.170 & 2.010 \\
\hline Relative Estimates & 0.510 & 0.607 & 0.557 & 0.517 & 0.737 & 1.697 & 3.900 & 6.700 \\
\hline Est-Std $\times 10^{-3}$ & 0.00155 & 0.0131 & 0.0753 & 0.136 & 0.209 & 0.569 & 1.290 & 2.170 \\
\hline RMSE $\times 10^{-3}$ & 0.147 & 0.119 & 0.153 & 0.199 & 0.223 & 0.606 & 1.556 & 2.763 \\
\hline \multicolumn{9}{|c|}{ Corwin and Schultz 2012} \\
\hline Estimates $\times 10^{-3}$ & & & -0.170 & -0.106 & -0.029 & 0.198 & 0.504 & 0.812 \\
\hline Relative Estimates & & & -0.567 & -0.353 & -0.097 & 0.660 & 1.680 & 2.707 \\
\hline Est-Std $\times 10^{-3}$ & & & 0.006 & 0.011 & 0.021 & 0.085 & 0.251 & 0.461 \\
\hline RMSE $\times 10^{-3}$ & & & 0.470 & 0.406 & 0.330 & 0.133 & 0.323 & 0.689 \\
\hline
\end{tabular}

There are 500 replications. There are 432000 periods, each of which represents one minute, in each replication. Data of each replication are generated according to the following system. The order flow is drawn from a binomial distribution, i.e. $B S_{t} \sim B(1,0.5)$. The mid-price return is influenced by the past order flow and a random shock drawn from a normal distribution of which the mean is zero and the variance is $4 \times 10^{-8}$. Thus there are the inventory control and the asymmetric information components in the spread. Formally, the mid-price returns are given by, $\Delta M_{t}=\frac{2}{3} B S_{t-1} \cdot \frac{S P_{t}}{2}+\varepsilon_{t}$ where $\varepsilon_{t} \sim N\left(0,4 \times 10^{-8}\right)$. The spread is fixed and equals to 0.0003 , i.e. $S P_{t}=0.0003$. The transaction price is the mid-price plus or minus a half spread, i.e. $s_{t}=M_{t}+\frac{S P_{t}}{2} \cdot B S_{t}$. Each replication is also sampled into longer time intervals: five-minute, fifteen-minute, thirty-minute, one-hour, four-hour, twelve-hour and twenty-four- hour, and only the close observations are kept. Thus, there are eight subgroups for each replication. For each subgroup, the standard deviation of mid-price returns, and the estimated spread are collected.

The other settings are the same as Table (1)

perform better in the combined case than in the pure price impact case, because the two biases offset one another (assuming that feedback trading is positive). 
1

2

3

4

5

9

10

11

12

13

14

15

16

17

18

19

20

21

22

23

24

25

26

27

28

29

30

31

32

33

34

35

36

37

38

39

40

41

42

43

44

45

46

47

48

49

50

51

52

53

54

55

56

57

58

59

60

61

62

63

64

65 


\section{Appendix}

\section{A Proof of Proposition (1)}

Definition 3 Let $A$ be a set of all conjectures of the true spread $A=\left\{\widetilde{S P}_{1}, \widetilde{S P}_{2}, \ldots, \widetilde{S P}_{n}\right\}$

Definition 4 Let $\mathrm{B}$ be a set of covariances of two adjacent conjectural mid-price returns obtained according to the conjecture of the true spread $B=\left\{\operatorname{Cov}_{1}, \operatorname{Cov}_{2}, \cdots, \operatorname{Cov}_{n}\right\}$, where $\operatorname{Cov}_{i}=\operatorname{Cov}\left[\widetilde{M}\left(\widetilde{S P_{i}}\right)_{t}, \widetilde{M}\left(\widetilde{S P}_{i}\right)_{t-1}\right]$.

One can find that sets $\mathrm{A}$ and $\mathrm{B}$ are one to one mapping.

Proposition (1): If there is no feedback trading, and no inventory control or asymmetric information components of the spread, then the spread and its conjecture, and thus the conjectural error, are serially independent or are fixed. If a conjecture of the spread $\widetilde{S P}_{i} \in A$ corresponds to $\operatorname{Cov}_{i}=\max (B)$, it equals the true spread i.e. $\widetilde{S P}_{i}=S P$.

Proof The covariance of two adjacent conjectures of mid-price returns is:

$$
\begin{aligned}
& \operatorname{Cov}\left(\Delta \widetilde{M}_{t}, \Delta \widetilde{M}_{t-1}\right) \\
= & E\left\{\left[\Delta \widetilde{M}_{t}-E\left(\Delta \widetilde{M}_{t}\right)\right]\left[\Delta \widetilde{M}_{t-1}-E\left(\Delta \widetilde{M}_{t-1}\right)\right]\right\}
\end{aligned}
$$

Assume the conjectural errors are fixed, expectations of errors are given by:

$$
E\left(\Omega_{t}\right)=E\left(\Omega_{t-1}\right)=E\left(\Omega_{t-2}\right)=\Omega
$$

and the exceptions of the multiplication of the conjectural errors are given by:

$$
E\left(\Omega_{t} \Omega_{t-1}\right)=E\left(\Omega_{t} \Omega_{t-2}\right)=E\left(\Omega_{t-1} \Omega_{t-2}\right)=\Omega^{2}
$$

Furthermore, assume the expectation of the conjectural mid-prices is zero. Thus, the covariance can be written as:

$$
\begin{aligned}
& \operatorname{Cov}\left(\Delta \widetilde{M}_{t}, \Delta \widetilde{M}_{t-1}\right) \\
= & E\left[\Delta \widetilde{M}_{t} \cdot \Delta \widetilde{M}_{t-1}\right] \\
= & E\left[\left(\Delta M_{t}+\frac{1}{2} \Omega B S_{t}-\frac{1}{2} \Omega B S_{t-1}\right)\left(\Delta M_{t-1}+\frac{1}{2} \Omega B S_{t-1}-\frac{1}{2} \Omega B S_{t-2}\right)\right] \\
= & E\left[\left(\Delta M_{t}+\frac{1}{2} \Omega B S_{t}-\frac{1}{2} \Omega B S_{t-1}\right) \Delta M_{t-1}\right. \\
& +\frac{1}{2} \Omega B S_{t-1}\left(\Delta M_{t}+\frac{1}{2} \Omega B S_{t}-\frac{1}{2} \Omega B S_{t-1}\right) \\
& \left.-\frac{1}{2} \Omega B S_{t-2}\left(\Delta M_{t}+\frac{1}{2} \Omega B S_{t}-\frac{1}{2} \Omega B S_{t-1}\right)\right] \\
= & E\left[\left(\Delta M_{t} \Delta M_{t-1}+\frac{1}{2} \Omega B S_{t} \Delta M_{t-1}-\frac{1}{2} \Omega B S_{t-1} \Delta M_{t-1}\right)\right. \\
& +\left(\Delta M_{t} \frac{1}{2} \Omega B S_{t-1}+\frac{1}{2} \Omega B S_{t} \frac{1}{2} \Omega B S_{t-1}-\frac{1}{2} \Omega B S_{t-1} \frac{1}{2} \Omega B S_{t-1}\right) \\
& \left.-\left(\Delta M_{t} \frac{1}{2} \Omega B S_{t-2}+\frac{1}{2} \Omega B S_{t} \frac{1}{2} \Omega B S_{t-2}-\frac{1}{2} \Omega B S_{t-1} \frac{1}{2} \Omega B S_{t-2}\right)\right]
\end{aligned}
$$

Re-arrange the equation further, we have:

$$
\begin{aligned}
= & E\left[\left(\Delta M_{t} \Delta M_{t-1}+\frac{1}{2} \Omega B S_{t} \Delta M_{t-1}-\frac{1}{2} \Omega B S_{t-1} \Delta M_{t-1}\right)\right. \\
& +\left(\frac{1}{2} \Delta M_{t} \Omega B S_{t-1}+\frac{1}{4} \Omega^{2} B S_{t} B S_{t-1}-\frac{1}{4} \Omega^{2} B S_{t-1}{ }^{2}\right) \\
& \left.-\left(\Delta M_{t} \frac{1}{2} \Omega B S_{t-2}+\frac{1}{4} \Omega^{2} B S_{t} B S_{t-2}-\frac{1}{4} \Omega^{2} B S_{t-1} B S_{t-2}\right)\right] \\
= & E\left(\Delta M_{t} \Delta M_{t-1}\right) \\
& +E\left(\frac{1}{2} \Omega B S_{t} \Delta M_{t-1}-\frac{1}{2} \Omega B S_{t-1} \Delta M_{t-1}+\frac{1}{2} \Delta M_{t} \Omega B S_{t-1}-\Delta M_{t} \frac{1}{2} \Omega B S_{t-2}\right. \\
& \left.+\frac{1}{4} \Omega^{2} B S_{t} B S_{t-1}-\frac{1}{4} \Omega^{2} B S_{t-1}{ }^{2}-\frac{1}{4} \Omega^{2} B S_{t} B S_{t-2}+\frac{1}{4} \Omega^{2} B S_{t-1} B S_{t-2}\right)
\end{aligned}
$$

Because the variable $B S$ is a binary variable $(1$ or -1$)$, then

$$
E\left(B S_{t-1}^{2}\right)=1
$$

Furthermore, because we assume there is no feedback trading, then:

$$
\begin{aligned}
& E\left(B S_{t} \Delta M_{t-1}\right)=0 \\
& E\left(B S_{t-1} \Delta M_{t-1}\right)=0
\end{aligned}
$$

Because we assume there is no IC\&AS components, then:

$$
\begin{aligned}
& E\left(B S_{t-1} \Delta M_{t}\right)=0 \\
& E\left(B S_{t-2} \cdot \Delta M_{t}\right)=0
\end{aligned}
$$

Equation (A.5) can be written as:

$$
\begin{aligned}
& \operatorname{Cov}\left(\Delta \widetilde{M}_{t}, \Delta \widetilde{M}_{t-1}\right) \\
= & E\left(\Delta M_{t} \Delta M_{t-1}\right) \\
& +E\left(\frac{1}{4} \Omega^{2} B S_{t} B S_{t-1}-\frac{1}{4} \Omega^{2}-\frac{1}{4} \Omega^{2} B S_{t} B S_{t-2}+\frac{1}{4} \Omega^{2} B S_{t-1} B S_{t-2}\right) \\
= & \operatorname{Cov}\left(\Delta M_{t} \cdot \Delta M_{t-1}\right)+\frac{1}{4} \Omega^{2} \cdot\left[2 E\left(B S_{t} \cdot B S_{t-1}\right)-E\left(B S_{t} \cdot B S_{t-2}\right)-1\right]
\end{aligned}
$$

The right hand side of the equation is a quadratic polynomial of the expectation of the error of the conjecture. For a given series, the first term on the right hand side is a constant. It is straightforward that when the expectation of the error is zero (i.e. 
$E(\Omega)=0$ ), the second term is zero. Furthermore, when $E(\Omega)=0$, there is a global extreme for the right hand side polynomial, symmetrically, the left hand side of the equation $\operatorname{Cov}\left(\Delta \widetilde{M}_{t}, \Delta \widetilde{M}_{t-1}\right)$ is also at the extreme value:

$$
\underset{\Omega}{\arg \max } \operatorname{Cov}\left(\Delta \widetilde{M}_{t}, \Delta \widetilde{M}_{t-1}\right)=0
$$

When the conjectural error is zero, the conjectural spread is the true spread:

$$
\Omega=S P-\widetilde{S P}_{i}=0
$$

Therefore the conjectural spread which maximises the covariance equals the true spread.

$$
\underset{\widetilde{S P}_{i} \in A}{\arg \max } \operatorname{Cov}\left(\Delta \widetilde{M}_{t}, \Delta \widetilde{M}_{t-1}\right)=S P
$$

Q.E.D.

\section{B Simplify equations (16) and (27)}

This section shows the detail of the simplification of equations (16) and (27). Feedback trading, inventory holding costs and asymmetric information costs are considered together.

Considering the inventory control and asymmetric information components of the spread, the true mid-price returns are given by:

$$
\Delta M_{t}=\frac{1}{2} \varrho S P B S_{t-1}+\epsilon_{t}
$$

The covariance of the two adjacent conjectural mid-price returns is given by,

$$
\begin{aligned}
& \operatorname{Cov}\left(\Delta \widetilde{M}_{t}, \Delta \widetilde{M}_{t-1}\right) \\
= & E\left\{\left[\Delta \widetilde{M}_{t}-E\left(\Delta \widetilde{M}_{t}\right)\right]\left[\Delta \widetilde{M}_{t-1}-E\left(\Delta \widetilde{M}_{t-1}\right)\right]\right\}
\end{aligned}
$$

Assume the expectation of conjectural mid-price returns to be zero. Thus the equation above can be written as:

$$
\begin{aligned}
& \operatorname{Cov}\left(\Delta \widetilde{M}_{t}, \Delta \widetilde{M}_{t-1}\right) \\
& =E\left[\Delta \widetilde{M}_{t} \cdot \Delta \widetilde{M}_{t-1}\right] \\
& =E\left\{\left[\frac{1}{2}(\varrho-1) \Omega B S_{t-1}+\frac{1}{2} \Omega B S_{t}+\frac{1}{2} \varrho \widetilde{S P}_{t-1} B S_{t-1}+\epsilon_{t}\right]\right. \\
& \left.\cdot\left[\frac{1}{2}(\varrho-1) \Omega B S_{t-2}+\frac{1}{2} \Omega B S_{t-1}+\frac{1}{2} \varrho \widetilde{S P}_{t-2} B S_{t-2}+\epsilon_{t-1}\right]\right\} \\
& =E\left\{\frac{1}{2}(-1+\varrho) \Omega B S_{t-2}\left[\frac{1}{2}(-1+\varrho) \Omega B S_{t-1}+\epsilon_{t}+\frac{1}{2} \Omega B S_{t}+\frac{1}{2} \varrho \widetilde{S P}_{t-1} B S_{t-1}\right]\right. \\
& +\epsilon_{t-1}\left[\frac{1}{2}(-1+\varrho) \Omega B S_{t-1}+\epsilon_{t}+\frac{1}{2} \Omega B S_{t}+\frac{1}{2} \varrho \widetilde{S P}_{t-1} B S_{t-1}\right] \\
& +\frac{1}{2} \Omega B S_{t-1}\left[\frac{1}{2}(-1+\varrho) \Omega B S_{t-1}+\epsilon_{t}+\frac{1}{2} \Omega B S_{t}+\frac{1}{2} \varrho \widetilde{S P}_{t-1} B S_{t-1}\right] \\
& \left.+\frac{1}{2} \varrho \widetilde{S P}_{t-2} B S_{t-2}\left[\frac{1}{2}(-1+\varrho) \Omega B S_{t-1}+\epsilon_{t}+\frac{1}{2} \Omega B S_{t}+\frac{1}{2} \varrho \widetilde{S P}_{t-1} B S_{t-1}\right]\right\} \\
& =E\left\{\left[\frac{1}{2} \cdot \frac{1}{2}(-1+\varrho) \Omega B S_{t-2}(-1+\varrho) \Omega B S_{t-1}+\frac{1}{2}(-1+\varrho) \Omega B S_{t-2} \epsilon_{t}\right.\right. \\
& \left.+\frac{1}{2} \cdot \frac{1}{2}(-1+\varrho) \Omega B S_{t-2} \Omega B S_{t}+\frac{1}{2} \cdot \frac{1}{2}(-1+\varrho) \Omega B S_{t-2} \varrho \widetilde{S P}_{t-1} B S_{t-1}\right] \\
& +\left[\frac{1}{2}(-1+\varrho) \Omega B S_{t-1} \epsilon_{t-1}+\epsilon_{t} \epsilon_{t-1}+\frac{1}{2} \Omega B S_{t} \epsilon_{t-1}+\frac{1}{2} \varrho \widetilde{S P}_{t-1} B S_{t-1} \epsilon_{t-1}\right] \\
& +\frac{1}{2} \cdot \frac{1}{2} \Omega B S_{t-1}(-1+\varrho) \Omega B S_{t-1}+\frac{1}{2} \Omega B S_{t-1} \epsilon_{t}+\frac{1}{2} \cdot \frac{1}{2} \Omega B S_{t-1} \Omega B S_{t} \\
& \left.+\frac{1}{2} \cdot \frac{1}{2} \Omega B S_{t-1} \varrho \widetilde{S P}_{t-1} B S_{t-1}\right]+\left[\frac{1}{2} \cdot \frac{1}{2} \varrho \widetilde{S P}_{t-2} B S_{t-2}(-1+\varrho) \Omega B S_{t-1}\right. \\
& \left.\left.+\frac{1}{2} \varrho \widetilde{S P}_{t-2} B S_{t-2} \epsilon_{t}+\frac{1}{2} \cdot \frac{1}{2} \varrho \widetilde{S P}_{t-2} B S_{t-2} \Omega B S_{t}+\frac{1}{2} \cdot \frac{1}{2} \varrho \widetilde{S P}_{t-2} B S_{t-2} \varrho \widetilde{S P}_{t-1} B S_{t-1}\right]\right\}
\end{aligned}
$$


Re-arrange the equation further, we have:

$$
\begin{aligned}
& \operatorname{Cov}\left(\Delta \tilde{M}_{t}, \Delta \widetilde{M}_{t-1}\right) \\
& =E\left\{\left[\frac{1}{4}(-1+\varrho)^{2} \Omega^{2} B S_{t-1} B S_{t-2}+\frac{1}{2}(-1+\varrho) \Omega B S_{t-2} \epsilon_{t}\right.\right. \\
& \left.+\frac{1}{4}(-1+\varrho) \Omega^{2} B S_{t} B S_{t-2}+\frac{1}{4} \varrho(-1+\varrho) \widetilde{S P} B S_{t-1} B S_{t-2} \Omega\right] \\
& +\left[\frac{1}{2}(-1+\varrho) \Omega B S_{t-1} \epsilon_{t-1}+\epsilon_{t} \epsilon_{t-1}+\frac{1}{2} \Omega B S_{t} \epsilon_{t-1}+\frac{1}{2} \varrho \widetilde{S P} B S_{t-1} \epsilon_{t-1}\right] \\
& +\frac{1}{4}(-1+\varrho) \Omega^{2} B S_{t-1}{ }^{2}+\frac{1}{2} \Omega B S_{t-1} \epsilon_{t}+\frac{1}{4} \Omega^{2} B S_{t} B S_{t-1} \\
& \left.+\frac{1}{4} \varrho \widetilde{S P} B S_{t-1}{ }^{2} \Omega\right]+\left[\frac{1}{4} \varrho(-1+\varrho) \Omega B S_{t-1} B S_{t-2} \widetilde{S P}+\frac{1}{2} \varrho \widetilde{S P} B S_{t-2} \epsilon_{t}\right] \\
& \left.\left.+\frac{1}{4} \varrho \widetilde{S P} \Omega B S_{t} B S_{t-2}+\frac{1}{4} \varrho^{2} \widetilde{S P}^{2} B S_{t-2} B S_{t-1}\right]\right\} \\
& =E\left(\epsilon_{t} \epsilon_{t-1}\right)+E\left[\frac{1}{4}(-1+\varrho)^{2} \Omega^{2} B S_{t-1} B S_{t-2}+\frac{1}{4}(-1+\varrho) \Omega^{2} B S_{t} B S_{t-2}\right. \\
& +\frac{1}{4}(-1+\varrho) \Omega^{2} B S_{t-1}^{2}+\frac{1}{4} \Omega^{2} B S_{t} B S_{t-1}+\frac{1}{2}(-1+\varrho) \Omega B S_{t-2} \epsilon_{t} \\
& +\frac{1}{2}(-1+\varrho) \Omega B S_{t-1} \epsilon_{t-1}+\frac{1}{2} \Omega B S_{t} \epsilon_{t-1}+\frac{1}{2} \Omega B S_{t-1} \epsilon_{t} \\
& +\frac{1}{4} \varrho(-1+\varrho) \widetilde{S P B S_{t-1}} B S_{t-2} \Omega+\frac{1}{4} \varrho \widetilde{S P B S} S_{t-1}^{2} \Omega \\
& +\frac{1}{4} \varrho(-1+\varrho) \Omega B S_{t-1} B S_{t-2} \widetilde{S P}+\frac{1}{4} \varrho \widetilde{S P} \Omega B S_{t} B S_{t-2} \\
& \left.+\frac{1}{2} \varrho \widetilde{S P} B S_{t-1} \epsilon_{t-1}+\frac{1}{2} \varrho \widetilde{S P} B S_{t-2} \epsilon_{t}+\frac{1}{4} \varrho^{2} \widetilde{S P}^{2} B S_{t-2} B S_{t-1}\right] \\
& =E\left(\epsilon_{t} \epsilon_{t-1}\right)+E\left\{\left[\frac{1}{4}(-1+\varrho)^{2} B S_{t-1} B S_{t-2}+\frac{1}{4}(-1+\varrho) B S_{t} B S_{t-2}\right.\right. \\
& \left.+\frac{1}{4}(-1+\varrho) B S_{t-1}{ }^{2}+\frac{1}{4} B S_{t} B S_{t-1}\right] \Omega^{2}+\left[\frac{1}{2}(-1+\varrho) B S_{t-2} \epsilon_{t}\right. \\
& \left.+\frac{1}{2}(-1+\varrho) B S_{t-1} \epsilon_{t-1}+\frac{1}{2} B S_{t} \epsilon_{t-1}+\frac{1}{2} B S_{t-1} \epsilon_{t}\right] \Omega \\
& +\left[\frac{1}{4}(-1+\varrho) B S_{t-1} B S_{t-2}+\frac{1}{4} B S_{t-1}^{2}\right. \\
& \left.+\frac{1}{4}(-1+\varrho) B S_{t-1} B S_{t-2}+\frac{1}{4} B S_{t} B S_{t-2}\right] \varrho \widetilde{S P} \Omega \\
& +\left[\frac{1}{2} B S_{t-1} \epsilon_{t-1}+\frac{1}{2} B S_{t-2} \epsilon_{t}\right] \varrho \widetilde{S P} \\
& \left.+\frac{1}{4} \varrho^{2} \widetilde{S P}^{2} B S_{t-2} B S_{t-1}\right\}
\end{aligned}
$$

Let following symbols to represent some parts of the equation above, because we assume order flows do not influence the midprice shocks following parts are zeros.

$$
E\left(B S_{t-2} \epsilon_{t}\right)=E\left(B S_{t-1} \epsilon_{t}\right)=0
$$

Because the variable BS is a binary variable and with a mean of zero, then:

$$
E\left(B S_{t-1}^{2}\right)=1
$$

$$
\begin{aligned}
\Pi_{0} & =E\left(B S_{t-1} B S_{t-2}\right) \\
\Pi_{1} & =E\left[\frac{1}{4}(-1+\varrho)^{2} B S_{t-1} B S_{t-2}+\frac{1}{4}(-1+\varrho) B S_{t} B S_{t-2}+\frac{1}{4}(-1+\varrho)+\frac{1}{4} B S_{t} B S_{t-1}\right] \\
& =\frac{1}{4} E\left[(-1+\varrho)^{2} \Pi_{0}+(-1+\varrho) B S_{t} B S_{t-2}+(-1+\varrho)+\Pi_{0}\right] \\
\Pi_{2} & =E\left[\frac{1}{2}(-1+\varrho) B S_{t-2} \epsilon_{t}+\frac{1}{2}(-1+\varrho) B S_{t-1} \epsilon_{t-1}+\frac{1}{2} B S_{t} \epsilon_{t-1}+\frac{1}{2} B S_{t-1} \epsilon_{t}\right] \\
& =\frac{1}{2} E\left[(-1+\varrho) B S_{t-1} \epsilon_{t-1}+B S_{t} \epsilon_{t-1}\right] \\
\Pi_{3} & =E\left[\frac{1}{4}(-1+\varrho) B S_{t-1} B S_{t-2}+\frac{1}{4} B S_{t-1}{ }^{2}+\frac{1}{4}(-1+\varrho) B S_{t-1} B S_{t-2}+\frac{1}{4} B S_{t} B S_{t-2}\right] \\
& =\frac{1}{4} E\left[2(-1+\varrho) \Pi_{0}+1+B S_{t} B S_{t-2}\right] \\
\Pi_{4} & =E\left(\frac{1}{2} B S_{t-1} \epsilon_{t-1}+\frac{1}{2} B S_{t-2} \epsilon_{t}\right) \\
& =\frac{1}{2} E\left(B S_{t-1} \epsilon_{t-1}\right)
\end{aligned}
$$

Substitute above equations into Equation (B.4), we have:

$$
\begin{aligned}
& \operatorname{Cov}\left(\Delta \widetilde{M}_{t}, \Delta \widetilde{M}_{t-1}\right) \\
= & E\left(\epsilon_{t} \epsilon_{t-1}\right)+\Pi_{1} \Omega^{2}+\Pi_{2} \Omega+\Pi_{3} \varrho \widetilde{S P} \Omega \\
& +\Pi_{4} \varrho \widetilde{S P}+\frac{1}{4} \varrho^{2} \widetilde{S P} \Pi_{0}
\end{aligned}
$$

Equation (B.8) suggests that when there are IC\&AS components and feedback trading, the covariance of the conjectural mid-price returns $\left(\operatorname{Cov}\left(\Delta \widetilde{M}_{t}, \Delta \widetilde{M}_{t-1}\right)\right)$ is no longer a function of conjectural errors $(\Omega)$ only but also a function of the conjecture of the spread $(\widetilde{S P})$. Furthermore, because the true spread $(S P)$ is certain for a given series, the conjectural errors $(\Omega)$ is a function of the conjecture of the spread. To investigate the relationship between the true spread and the conjecture of it, we re-arrange the equation to make $\widetilde{S P}$ be the only variable of the equation. Replace the conjectural error $(\Omega)$ by the true spread $(S P)$ and the conjectural spread $(\widetilde{S P})$, 
we have:

$$
\begin{aligned}
& \operatorname{Cov}\left(\Delta \widetilde{M}_{t}, \Delta \widetilde{M}_{t-1}\right) \\
= & E\left(\epsilon_{t} \epsilon_{t-1}\right)+\Pi_{1}(S P-\widetilde{S P})^{2}+\Pi_{2}(S P-\widetilde{S P}) \\
& +\Pi_{3}\left[\varrho \widetilde{S P}(S P-\widetilde{S P})+\Pi_{4} \varrho \widetilde{S P}+\frac{1}{4} \Pi_{0} \varrho^{2} \widetilde{S P}^{2}\right. \\
= & E\left(\epsilon_{t} \epsilon_{t-1}\right)+\Pi_{1} S P^{2}-2 \Pi_{1} \widetilde{S P S P}+\Pi_{1} \widetilde{S P}^{2}+\Pi_{2} S P \\
& -\Pi_{2} \widetilde{S P}+\Pi_{3} \varrho \widetilde{S P S P}-\Pi_{3} \varrho \widetilde{S P}^{2}+\Pi_{4} \varrho \widetilde{S P}+\frac{1}{4} \varrho^{2} \widetilde{S P}^{2} \Pi_{0} \\
= & E\left(\epsilon_{t} \epsilon_{t-1}\right)+\Pi_{1} S P^{2}+\Pi_{2} S P-2 \Pi_{1} S P S P-\Pi_{2} \widetilde{S P} \\
& +\Pi_{3} \widehat{S P S P}+\Pi_{4} \varrho \widetilde{S P}+\Pi_{1} \widetilde{S P}^{2}+\frac{1}{4} \varrho^{2} \widetilde{S P}^{2} \Pi_{0}-\Pi_{3} \varrho \widetilde{S P}^{2} \\
= & \left(\Pi_{1}+\frac{1}{4} \varrho^{2} \Pi_{0}-\varrho \Pi_{3}\right) \cdot \widetilde{S P}^{2}+\left[\varrho \Pi_{3} \cdot S P+\varrho \Pi_{4}-2 \Pi_{1} \cdot S P-\Pi_{2}\right] \\
& \cdot \widetilde{S P}+E\left(\epsilon_{t} \cdot \epsilon_{t-1}\right)+\Pi_{1} \cdot S P^{2}+\Pi_{2} \cdot S P
\end{aligned}
$$

We now discuss possible errors that if we still let $(S P-\Omega)$ which maximises the covariance between two adjacent conjectures of mid-price returns $\operatorname{Cov}\left(\Delta \widetilde{M}_{t}, \Delta \widetilde{M}_{t-1}\right)$ to be the estimate of the true spread. Thus, the estimate is given by:

$$
\widehat{S P}=-\left[\frac{-2 \Pi_{1} S P-\Pi_{2}+\Pi_{3} S P \varrho+\Pi_{4} \varrho}{2\left(\Pi_{1}+\frac{1}{4} \Pi_{0} \varrho^{2}-\Pi_{3} \varrho\right)}\right]
$$

where $\widehat{S P}$ is the value of $(S P-\Omega)$ which maximises $\operatorname{Cov}\left(\Delta \widetilde{M}_{t}, \Delta \widetilde{M}_{t-1}\right)$, and is the estimate of the true spread. When there are no IC\&AS components of the spread $(\varrho=0)$, no feedback trading $\left(\Pi_{2}=\Pi_{4}=0\right)$, and no autocorrelated order flows $\left(\Pi_{o}=0\right.$ and $\Pi_{1}=-1$ and $\left.\Pi_{3}=1\right)$, the equation above becomes:

$$
\widehat{S P}=S P
$$

and thus,

$$
\Omega=0
$$

The equations above suggest that under the ideal conditions, Equation (B.10) reduces to the simple version of the estimator and in this circumstance, the estimator is unbiased.

\section{Errors of the estimator}

\section{C.1 Feedback trading}

Assume there is feedback trading and there are no inventory control and asymmetric information components of the spread, thus $\varrho=0$, then Equation (B.8) becomes,

$$
\begin{aligned}
& \operatorname{Cov}\left(\Delta \widetilde{M}_{t}, \Delta \widetilde{M}_{t-1}\right) \\
= & E\left(\Delta \widetilde{M}_{t} \cdot \Delta \widetilde{M}_{t-1}\right) \\
= & E\left(\epsilon_{t} \cdot \epsilon_{t-1}\right)+\Pi_{1} \cdot \Omega^{2}+\Pi_{2} \cdot \Omega
\end{aligned}
$$

where

$$
\begin{aligned}
& \Pi_{0}=E\left(B S_{t-1} \cdot B S_{t-2}\right) \\
& \Pi_{1}=\frac{1}{4} E\left[\Pi_{0}-B S_{t} \cdot B S_{t-2}-1+\Pi_{0}\right] \\
& \Pi_{2}=\frac{1}{2} E\left[-B S_{t-1} \cdot \epsilon_{t-1}+B S_{t} \cdot \epsilon_{t-1}\right]
\end{aligned}
$$

Because the covariance of order flows are usually very small compare to 1 , it is safe to take approximation that let $\Pi_{1}=-\frac{1}{4}$. From (C.2) into Equation (B.10), it becomes:

$$
\begin{aligned}
& \widehat{S P} \\
= & -\frac{-2 \Pi_{1} S P-\Pi_{2}}{2\left(\Pi_{1}\right)} \\
= & S P+\frac{\Pi_{2}}{2 \Pi_{1}} \\
= & S P+\frac{\frac{1}{2} E\left(B S_{t} \epsilon_{t-1}-B S_{t-1} \epsilon_{t-1}\right)}{-2 \cdot \frac{1}{4}} \\
= & S P+E\left(B S_{t-1} \epsilon_{t-1}\right)-E\left(B S_{t} \epsilon_{t-1}\right)
\end{aligned}
$$

\section{C.2 Inventory Control and Asymmetric Information Components}

Assume there are inventory control and asymmetric information components of the spread and there is no feedback trading, then Equation (B.8) becomes,

$$
\begin{aligned}
& \operatorname{Cov}\left(\Delta \widetilde{M}_{t}, \Delta \widetilde{M}_{t-1}\right) \\
= & E\left(\Delta \widetilde{M}_{t} \cdot \Delta \widetilde{M}_{t-1}\right) \\
= & E\left(\epsilon_{t} \cdot \epsilon_{t-1}\right)+\Pi_{1} \cdot \Omega^{2}+\varrho \Pi_{3} \cdot(S P-\Omega) \cdot \Omega \\
& +\frac{1}{4} \varrho^{2} \Pi_{0} \cdot\left[(S P-\Omega)^{2}\right]
\end{aligned}
$$

where

$$
\begin{aligned}
& \Pi_{0}=E\left(B S_{t-1} \cdot B S_{t-2}\right) \\
& \Pi_{1}=\frac{1}{4} E\left[(\varrho-1)^{2} \Pi_{0}+(\varrho-1) B S_{t} \cdot B S_{t-2}+(\varrho-1)+\Pi_{0}\right] \\
& \Pi_{3}=\frac{1}{4} E\left[1+2(\varrho-1) \Pi_{0}+B S_{t} \cdot B S_{t-2}\right]
\end{aligned}
$$


And Equation (B.10) becomes:

$$
\widehat{S P}=-\frac{-2 \Pi_{1} S P+\Pi_{3} S P \varrho}{2\left(\Pi_{1}+\frac{1}{4} \Pi_{0} \varrho^{2}-\Pi_{3} \varrho\right)}=\frac{\left(2 \Pi_{1}-\Pi_{3} \varrho\right)}{2\left(\Pi_{1}+\frac{1}{4} \Pi_{0} \varrho^{2}-\Pi_{3} \varrho\right)} \cdot S P
$$

The numerator of Equation (C.6) can be simplified:

$$
\begin{aligned}
& \left(2 \Pi_{1}-\Pi_{3} \varrho\right) \\
= & 2(-1+\varrho)^{2} \Pi_{0}+2(-1+\varrho) B S_{t} B S_{t-2} \\
& +2(-1+\varrho)+2 \Pi_{0}-2(-1+\varrho) \Pi_{0} \varrho-\varrho-B S_{t} B S_{t-2} \varrho \\
= & 2(-1+\varrho)^{2} \Pi_{0}+2 \Pi_{0}-2(-1+\varrho) \Pi_{0} \varrho \\
& +2(-1+\varrho) B S_{t} B S_{t-2}-B S_{t} B S_{t-2} \varrho \\
& +2(-1+\varrho)-\varrho \\
= & 2 \Pi_{0} \varrho^{2}-4 \Pi_{0} \varrho+4 \Pi_{0}-2 \Pi_{0} \varrho^{2}+2 \Pi_{0} \varrho \\
& +2 \varrho B S_{t} B S_{t-2}-2 B S_{t} B S_{t-2}-B S_{t} B S_{t-2} \varrho \\
& -2+\varrho \\
= & 4 \Pi_{0}-2 \Pi_{0} \varrho+\varrho B S_{t} B S_{t-2}-2 B S_{t} B S_{t-2}-2+\varrho \\
= & 4 B S_{t-1} B S_{t-2}-2 B S_{t} B S_{t-2}-2+\left(B S_{t} B S_{t-2}+1-2 B S_{t-1} B S_{t-2}\right) \varrho
\end{aligned}
$$

The dominator of Equation (C.6) can be simplified:

$$
\begin{aligned}
& 2\left(\Pi_{1}+\Pi_{0} \varrho^{2}-\Pi_{3} \varrho\right) \\
= & 2\left(\left[(-1+\varrho)^{2} \Pi_{0}+(-1+\varrho) B S_{t} B S_{t-2}+(-1+\varrho)+\Pi_{0}\right]\right. \\
& \left.+\Pi_{0} \varrho^{2}-\left[2(-1+\varrho) \Pi_{0}+1+B S_{t} B S_{t-2}\right] \varrho\right) \\
= & 2\left(\Pi_{0} \varrho^{2}-2 \Pi_{0} \varrho+\Pi_{0}+(-1+\varrho) B S_{t} B S_{t-2}-1+\varrho+\Pi_{0}\right. \\
& \left.+\Pi_{0} \varrho^{2}+2(1-\varrho) \Pi_{0} \varrho-\varrho-B S_{t} B S_{t-2} \varrho\right) \\
= & 2\left(2 B S_{t-1} B S_{t-2}-B S_{t} B S_{t-2}-1\right) \\
& +2\left(\Pi_{1}+\Pi_{0} \varrho^{2}-\Pi_{3} \varrho\right) \\
= & 2\left(\left[(-1+\varrho)^{2} \Pi_{0}+(-1+\varrho) B S_{t} B S_{t-2}+(-1+\varrho)+\Pi_{0}\right]\right. \\
& \left.+\Pi_{0} \varrho^{2}-\left[2(-1+\varrho) \Pi_{0}+1+B S_{t} B S_{t-2}\right] \varrho\right) \\
= & 2\left(\Pi_{0} \varrho^{2}-2 \Pi_{0} \varrho+\Pi_{0}+(-1+\varrho) B S_{t} B S_{t-2}-1+\varrho+\Pi_{0}\right. \\
& \left.+\Pi_{0} \varrho^{2}+2(1-\varrho) \Pi_{0} \varrho-\varrho-B S_{t} B S_{t-2} \varrho\right) \\
= & 2\left(2 B S_{t-1} B S_{t-2}-B S_{t} B S_{t-2}-1\right)
\end{aligned}
$$

Substitute the results of the simplification back to Equation (C.6), the equation becomes,

$$
\begin{aligned}
& \widehat{S P} \\
= & \frac{\left(2 \Pi_{1}-\Pi_{3} \varrho\right)}{2\left(\Pi_{1}+\frac{1}{4} \Pi_{0} \varrho^{2}-\Pi_{3} \varrho\right)} \cdot S P \\
= & \frac{3 B S_{t-1} B S_{t-2}-2 B S_{t} B S_{t-2}-2+\left(B S_{t} B S_{t-2}+1-2 B S_{t-1} B S_{t-2}\right) \varrho}{\left(4 B S_{t-1} B S_{t-2}-2 B S_{t} B S_{t-2}-2\right)} \cdot S P \\
= & {\left[1+\frac{\left(B S_{t} B S_{t-2}+1-2 B S_{t-1} B S_{t-2}\right) \varrho}{\left(4 B S_{t-1} B S_{t-2}-2 B S_{t} B S_{t-2}-2\right)}\right] \cdot S P } \\
= & \left(1-\frac{1}{2} \varrho\right) \cdot S P
\end{aligned}
$$




\section{References}

Bleaney, M. and Z. Li (2015). The performance of bid-ask spread estimators under less than ideal conditions. Stud Econ Financ Forthcoming.

Choi, J. Y., D. Salandro, and K. Shastri (1988). On the estimation of bid-ask spreads: Theory and evidence. J Financ Quant Anal 23(2): 219-230.

Corwin, S. A. and P. Schultz, P. (2012). A simple way to estimate bid-ask spreads from daily high and low prices. J Financ 67(2): 719-760.

Daníelsson, J. and R. Love (2006). Feedback trading. Int J Financ Econ 11(1): 35-53.

De Long, J. B., A. Shleifer, L. H. Summers, and R. J. Waldmann (1990). Positive feedback investment strategies and destabilizing rational speculation. J Financ 45(2): 379-395.

Dean, W. G. and R. W. Faff (1990). Evidence of feedback trading with Markov switching regimes. Rev Quant Financ Account 30(2): 133-151.

Evans, M. D. D. and R. K. Lyons (2002). Order flow and exchange rate dynamics. J Political Econ 110(1): 170-180.

George, T. J., G. Kaul, and M. Nimalendran (1991). Estimation of the bid-ask spread and its components: A new approach. Rev Financ Stud 4(4): 623-656.

Glosten, L. and P. Milgrom (1985). Bid ask and transaction prices in a specialist market with heterogeneously informed trades. J Financ Econ 14(1): 71-100.

Glosten, L. R. and L. E. Harris (1988). Estimating the components of the bid/ask spread. J Financ Econ 21(1): 123-142.

Goyenko, R. Y., C. W. Holden, and C. A. Trzcinka (2009). Do liquidity measures measure liquidity? J Financ Econ 92(2): 153 - 181.

Hasbrouck, J. (1991). Measuring the information content of stock trades. J Financ 46(1): 179-207.

Hasbrouck, J. (2004). Liquidity in the futures pits: Inferring market dynamics from incomplete data. J Financ Quant Anal 39(2): 305-326.

Hasbrouck, J. (2009). Trading costs and returns for u.s. equities: Estimating effective costs from daily data. J Financ 64(3): 14451477.

Holden, C. W. (2009). New low-frequency spread measures. J Financ Markets 12(4): $778-813$.

Huang, R. D. and H. R. Stoll (1997). The components of the bid-ask spread: A general approach. Rev Financ Stud 10(4): 995-1034.

Kyle, A. S. (1985). Continuous auctions and insider trading. Econometrica 53(6): 1315-1335.

Laux, P. A., and A. J. Senchack, , Jr. (1994). Estimating the bid-ask spread in a heteroskedastic market: The case of foreign currency futures. Rev Quant Financ Account 4(3): pp. 219-237.

Lesmond, D. A., J. P. Ogden, and C. A. Trzcinka (1999). A new estimate of transaction costs. Rev Financ Stud 12(5): pp. $1113-1141$.

Lin, C. H., H Hsu, and C. Y. Chiang (2005). Trading patterns and performance of trader types in taiwan futures market. Rev Pac Basin Financ Markets Policies 12 8(2): $217-234$.

Nofsinger, J. R. and R. W. Sias (1999). Herding and feedback trading by institutional and individual investors. J Financ 54(6): $2263-2295$.

Roll, R. (1984). A simple implicit measure of the effective bid-ask spread in an efficient market. J Financ 39(4): 1127-1139.

Stoll, H. R. (1989). Inferring the components of the bid-ask spread: Theory and empirical tests. J Financ 44(1): 115-134. 